\title{
A Straightforward and Highly Efficient Catalyst-Free One-Pot Synthesis of Dithiocarbamates Under Solvent Free Conditions
}

Najmedin Azizi, ${ }^{b}$ Fezzeh Arynasab ${ }^{a}$ and Mohammad R. Saidi*a

${ }^{a}$ Department of Chemistry, Sharif University of Technology, P. O. Box 114659516 Tehran 11365, Iran

${ }^{b}$ Chemistry and Chemical Engineering Research Center of Iran, P.O. Box 14335-186, Tehran, Iran

Emial: saidi@sharif.edu

\section{Table of Content}

Experimental procedure: .S2

Copy of ${ }^{1} \mathrm{H}$ and ${ }^{13} \mathrm{C}$ NMR S3-S31 


\section{General}

NMR spectra were recorded on a Bruker ACF 500 using $\mathrm{CDCl}_{3} / \mathrm{CCl}_{4}$ or $\mathrm{CDCl}_{3} / \mathrm{DMSO}-\mathrm{d}^{6}$ as solvent. Column chromatography was performed on silica gel, Merck grade 60. Ethyl acetate, petroleum ether and water were distilled before use. Alkyl halides and amines and other chemical were purchased and used without further purification.

\section{General procedure for one-pot synthesis of dithiocarbamates}

A ice cold mixture of the organic halide (3 mmol) and carbon disulfide (6 mmol) in the test tube, amine (6 mmol) was added and stirred at $0{ }^{\circ} \mathrm{C}$ for 30 min, then warmed to room temperate and stirring was continued until the reaction was complete (reaction mixture solidified or monitored by TLC). The reaction was quenched by addition of water and was extracted with EtOAc, dried over anhyd. $\mathrm{Na}_{2} \mathrm{SO}_{4}$, and evaporated to give NMR pure product. Further purification in some cases can be achieved by silica gel column chromatography eluted with petroleum ether and ethyl acetate or recrystallized to afford the pure dithiocarbamate. 

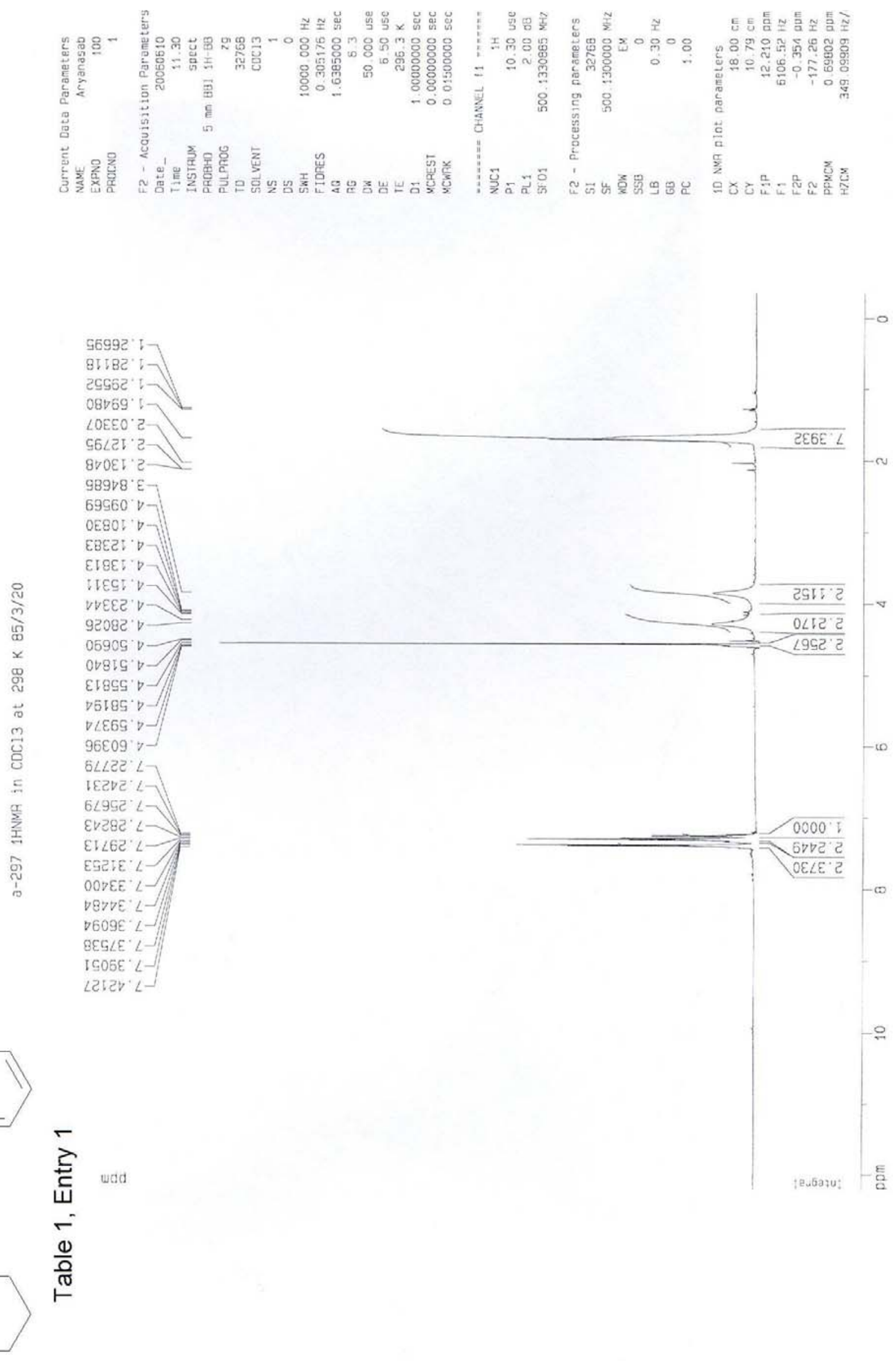


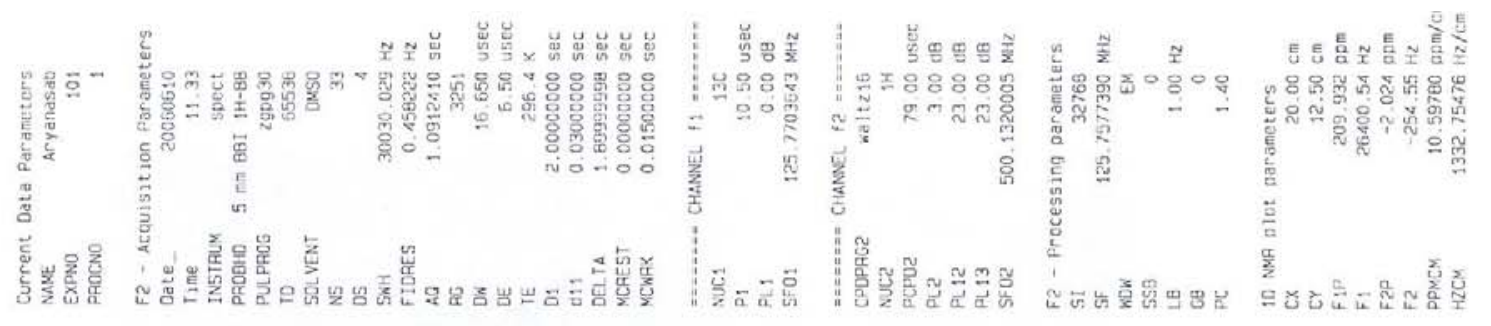

$159^{\circ} 95$
$998 \times 2-7$

$635^{\circ} 2 V$

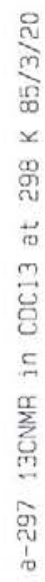

$\{1$

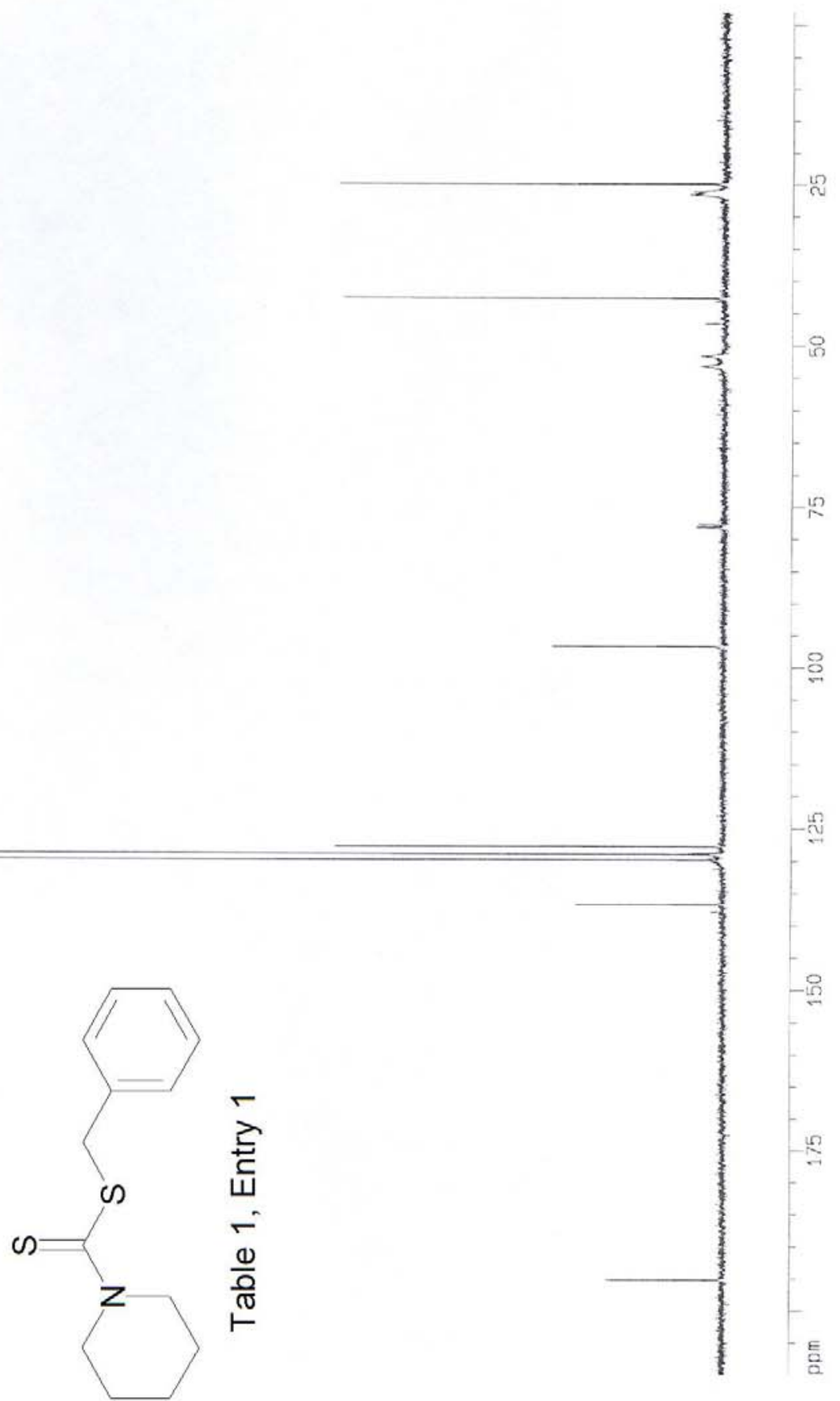




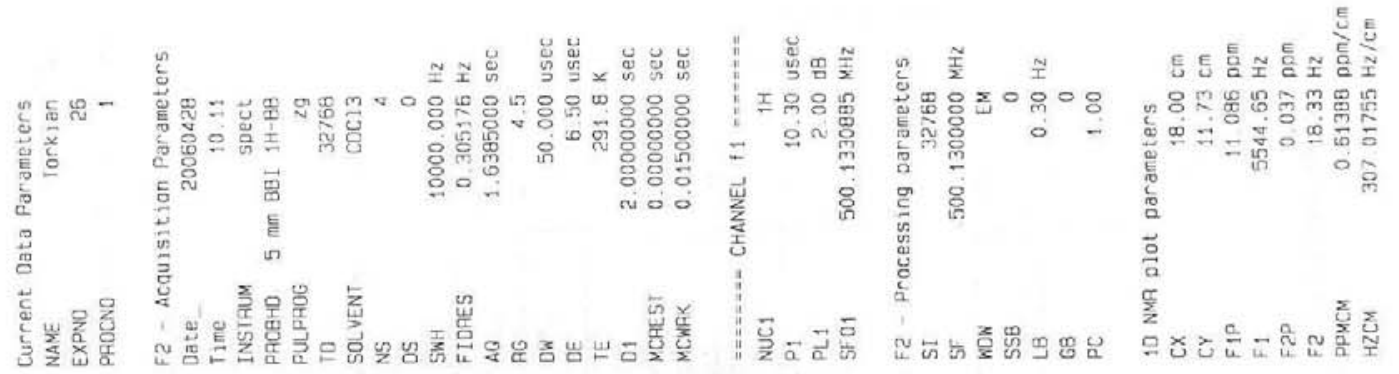

99895

$0+9 \angle 9$

$\angle B \angle 09^{\circ}$

OEITI

SELI

$\angle I \angle 29^{\circ}$

टBSE6 $1-$

$\angle I E 6 \angle{ }^{\circ} \varepsilon-$

$00888 \varepsilon-$

$\varepsilon 6688^{\prime} \varepsilon-$

S0206 $E-$

$\neg 6 E 06^{\circ} \varepsilon-$

$8918 \mathrm{I}^{\circ} \mathrm{C}$

ОВटह0 9

ODDEO $\mathrm{C}-$

टाLEO 97

E6590 9

$\angle \nabla \nabla G 0^{\circ} \mathrm{G}$

$87990^{\circ} \mathrm{G}$

5298 9

00886 ' $\mathrm{s}$

6706! 9

จर्डा 2

00555.

$6902 \mathrm{C}^{\circ} \mathrm{-}$

ILEO8 9 -

$18 \angle 18^{\circ} \mathrm{S}_{-}$

$\checkmark \angle E Z 8^{\circ} \mathrm{G}-$

GLLER G-

$\varepsilon 9 \angle 98^{\circ} \mathrm{S}^{-}$
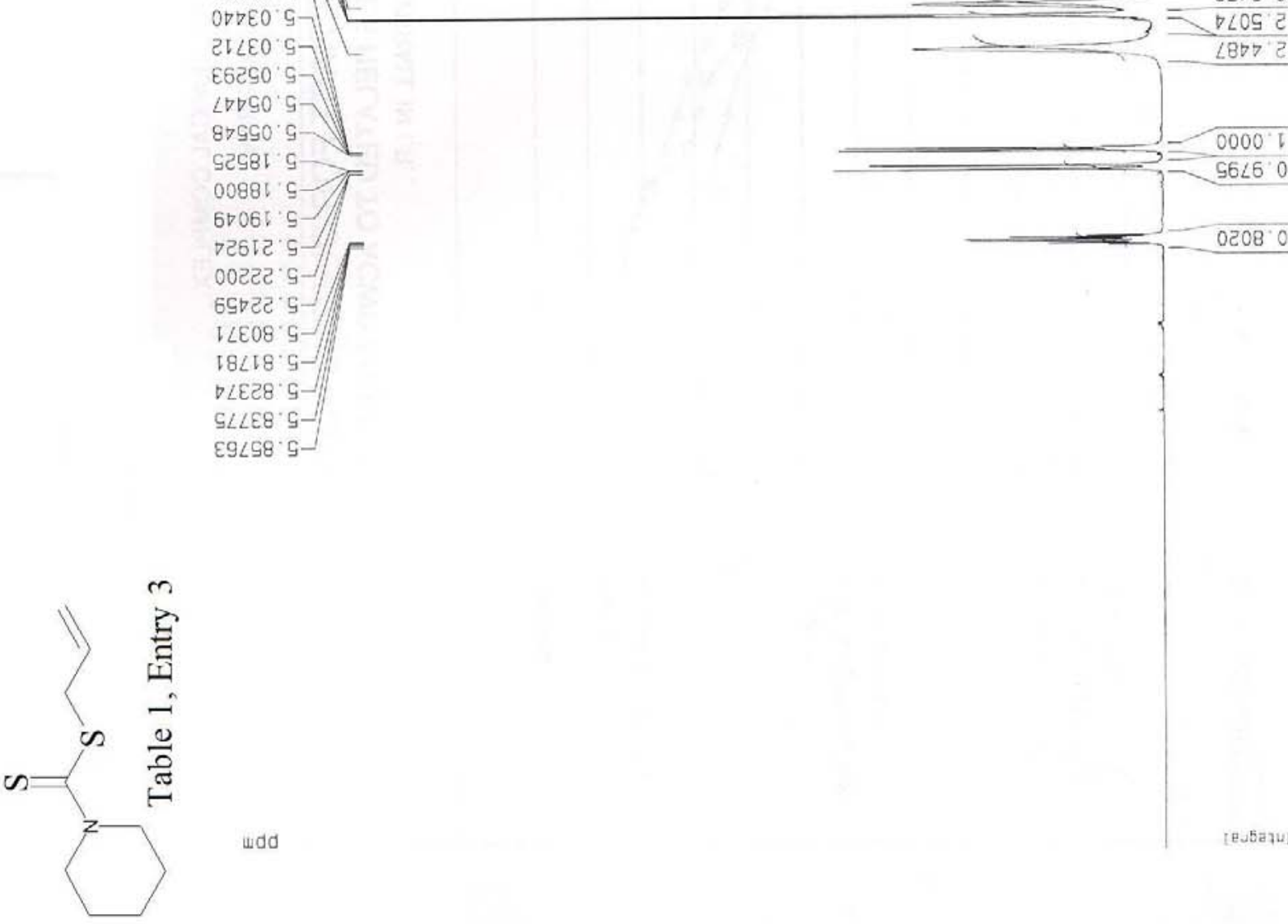


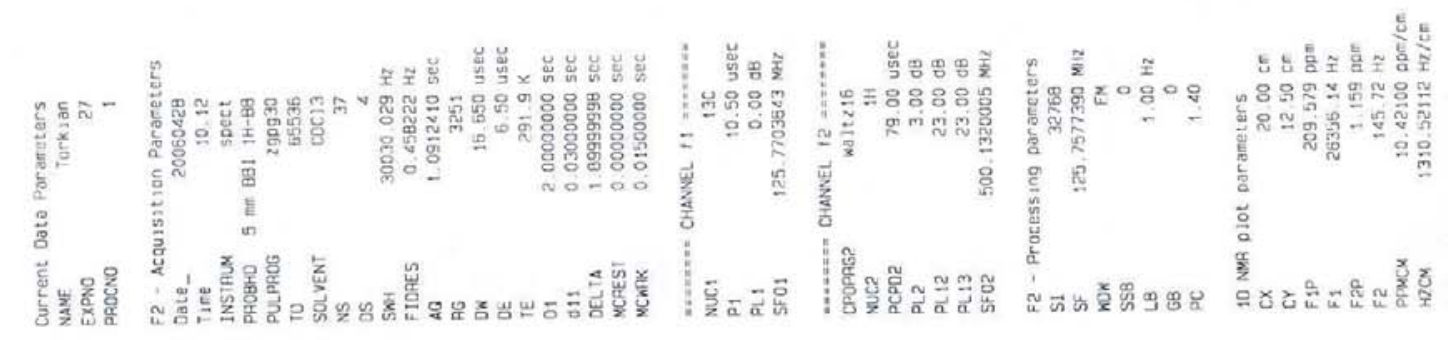

$\varepsilon 89^{\circ} \nabla \mathcal{C}$

$088^{\prime} \mathrm{GC}$

$0 \angle \nabla^{\circ} 92$

$199^{\circ}$ or

ISI IS
SL2.ES

$66 L^{\circ} \angle L$

$990^{\circ} 8 L$

$60 \varepsilon^{\circ} B L$

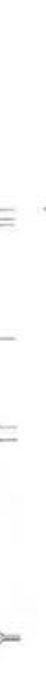

$20 L B L$

$692 \cdot \varepsilon E \digamma-$
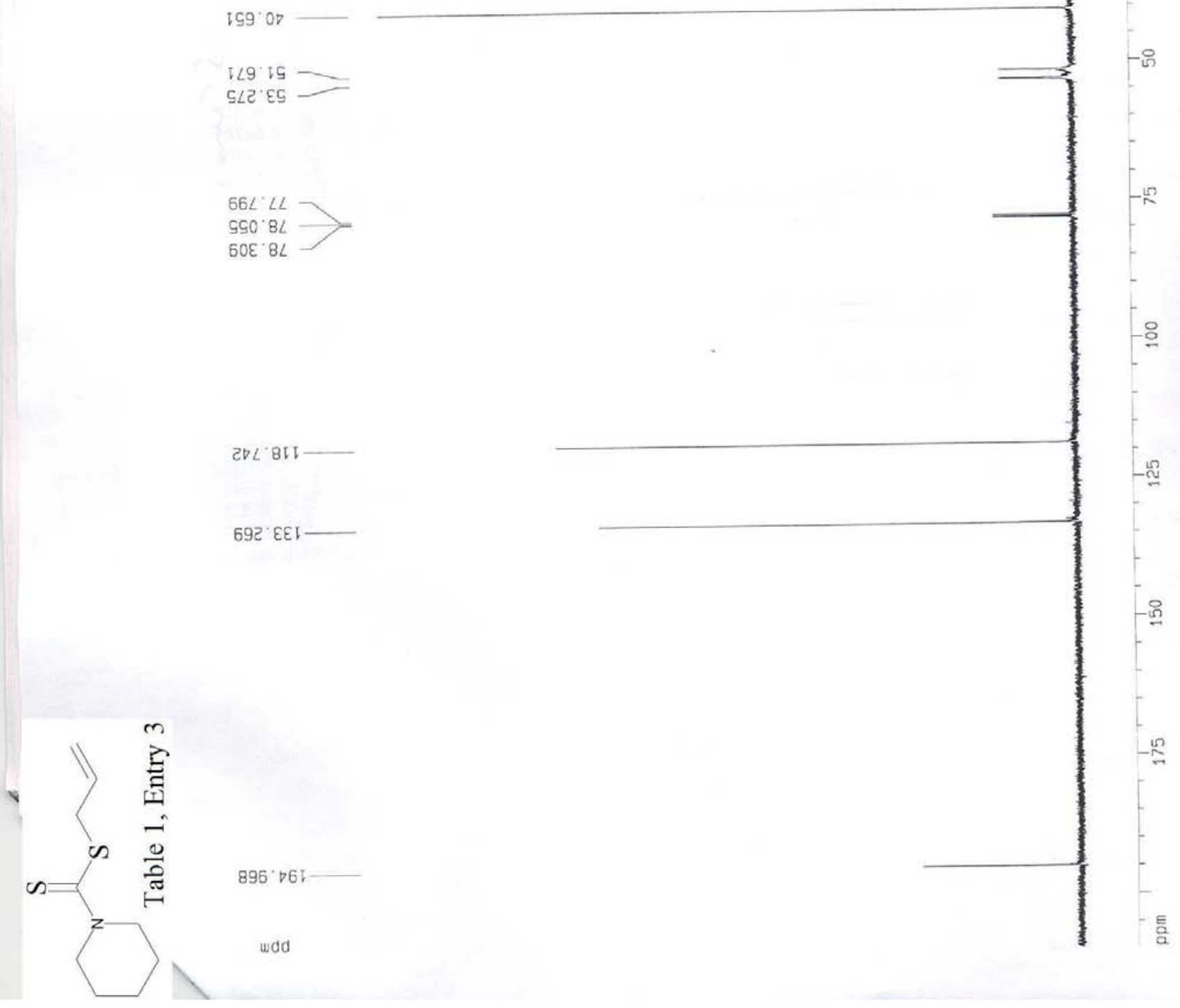

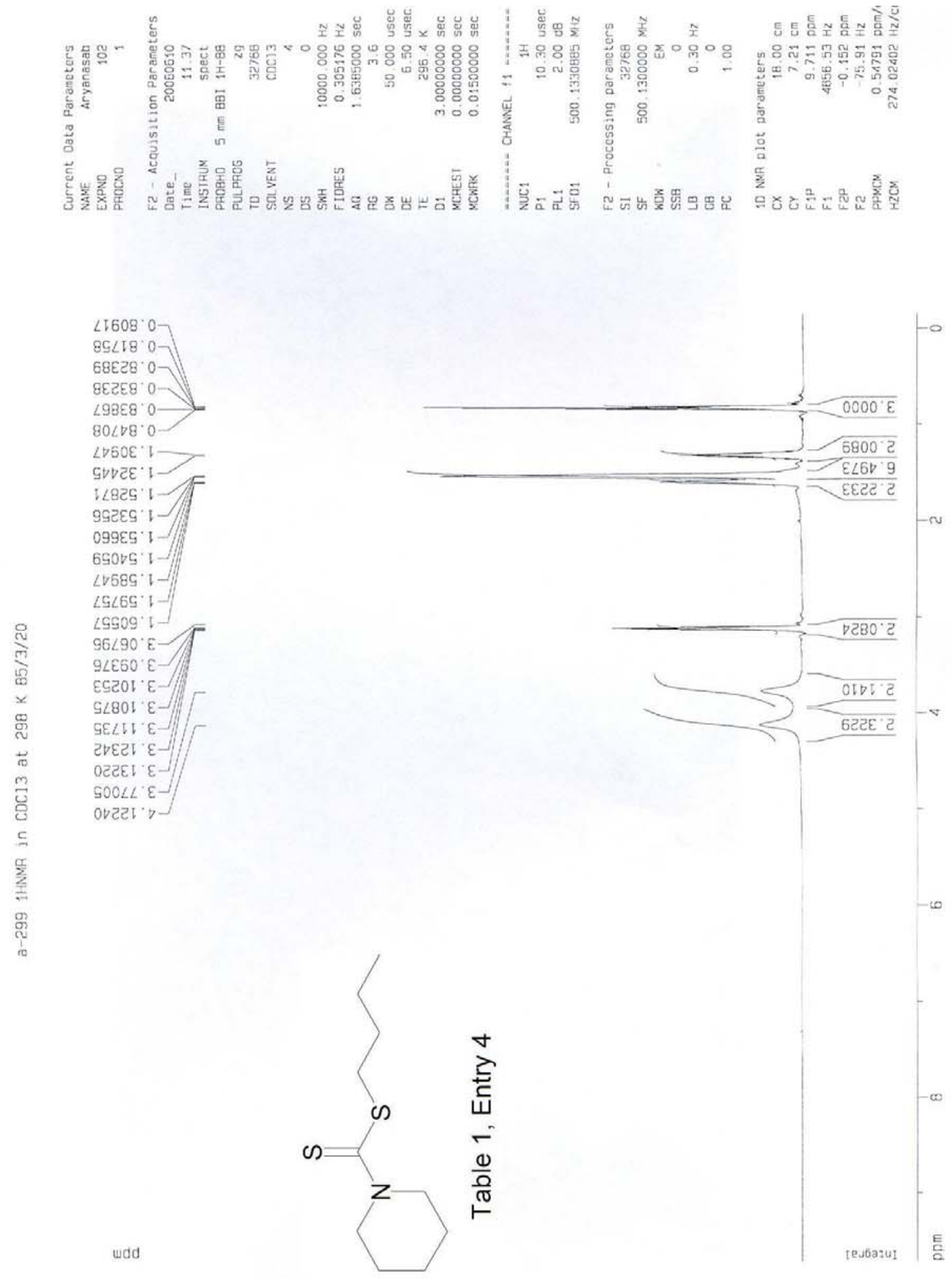

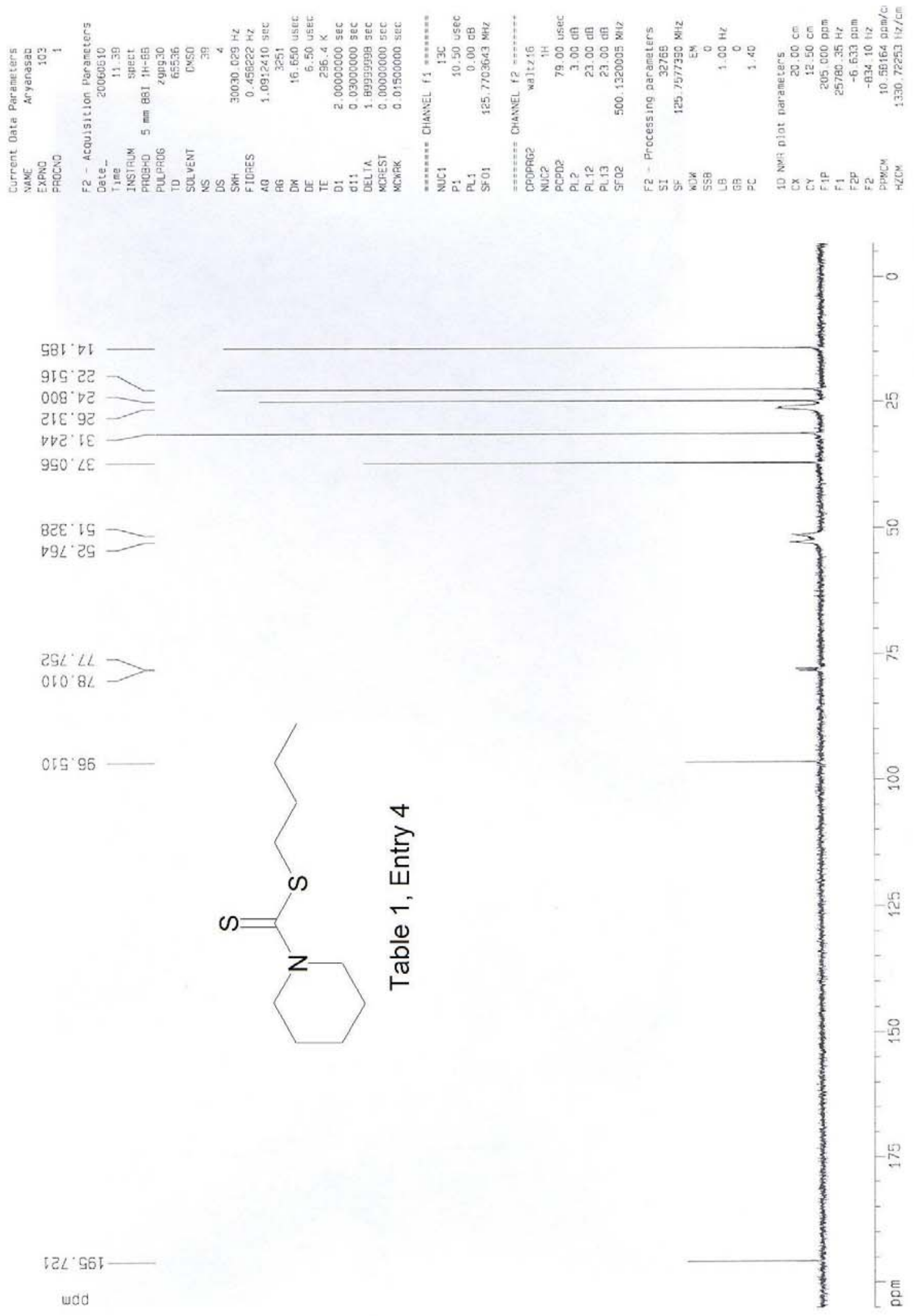

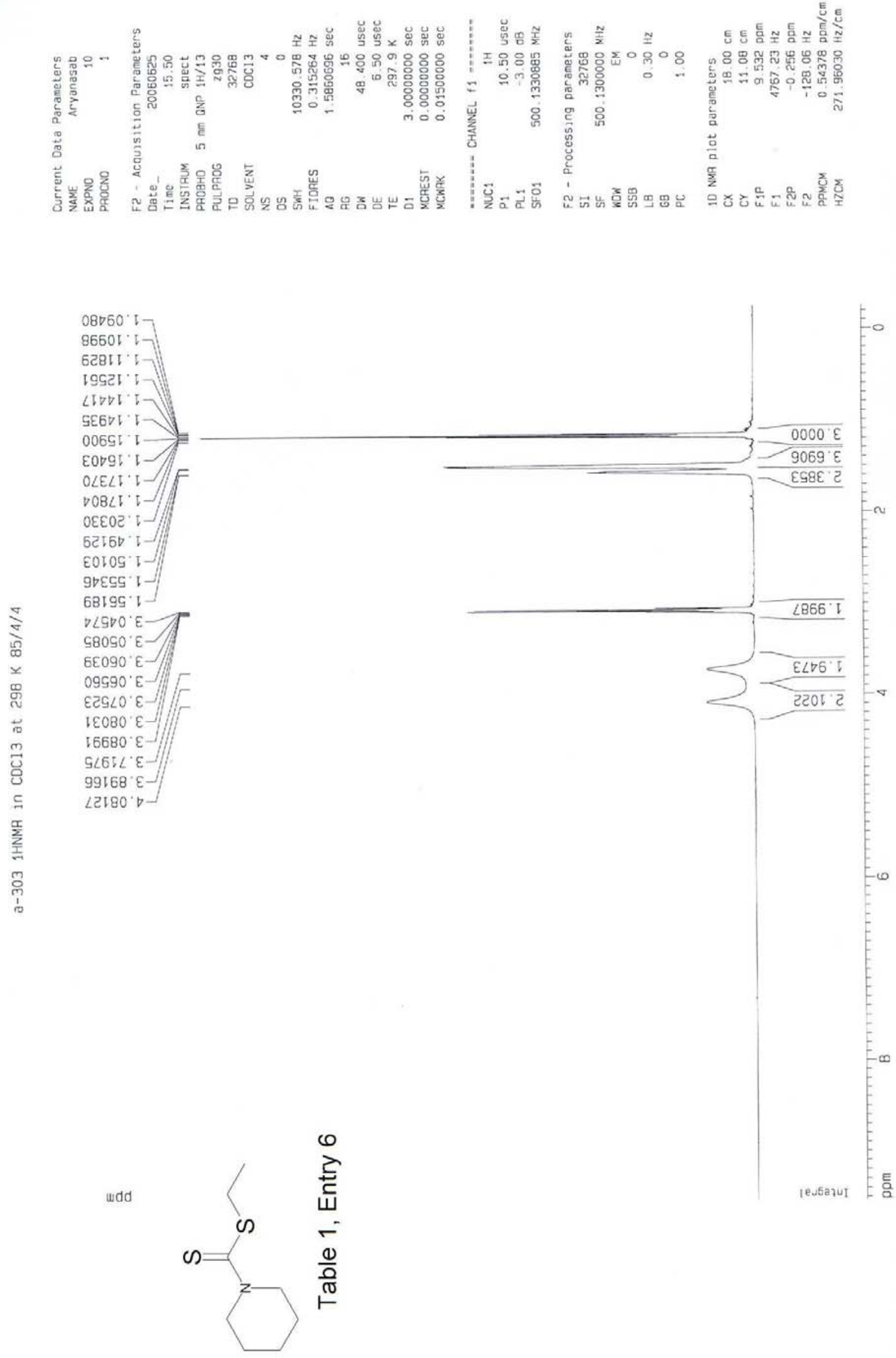

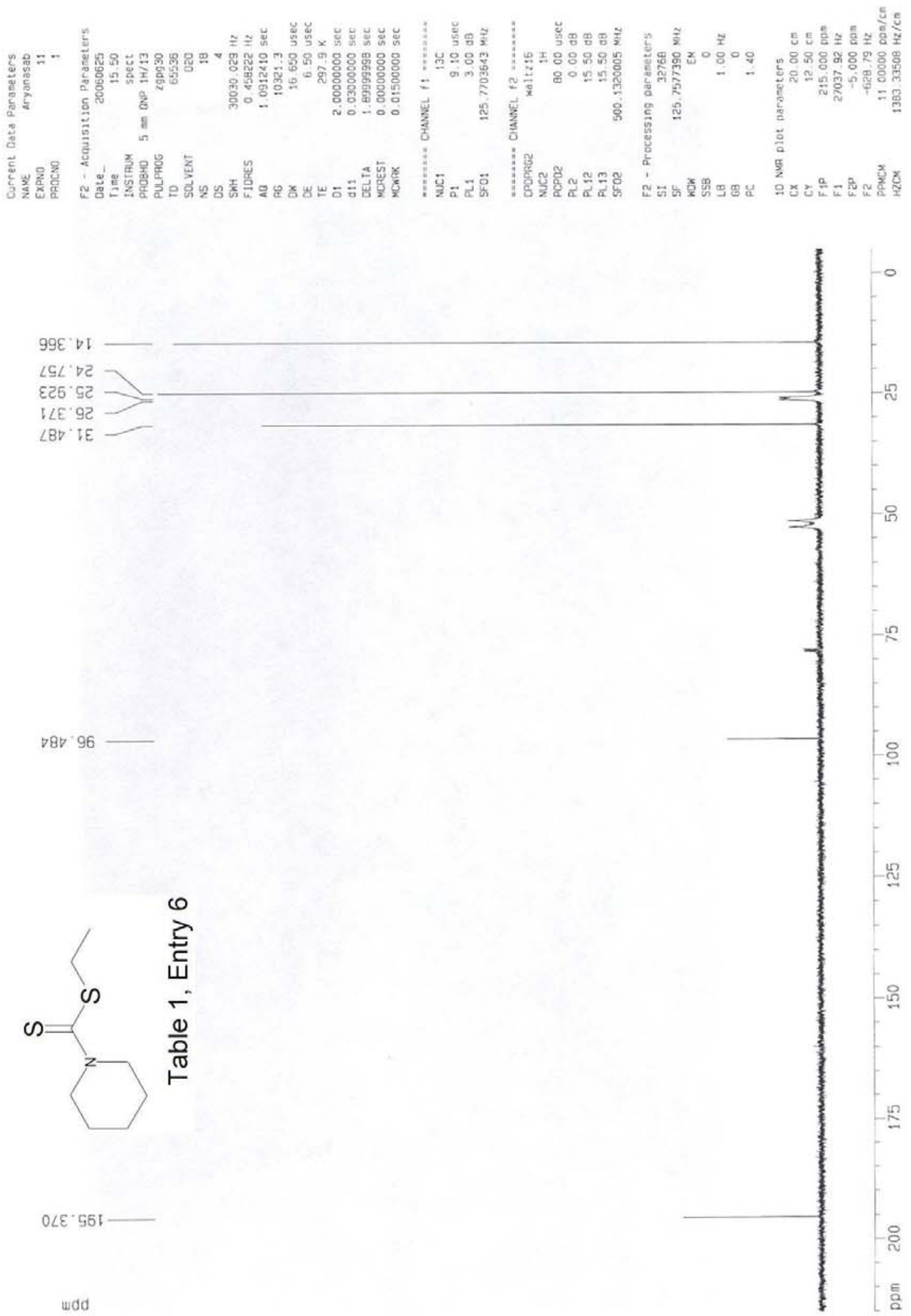

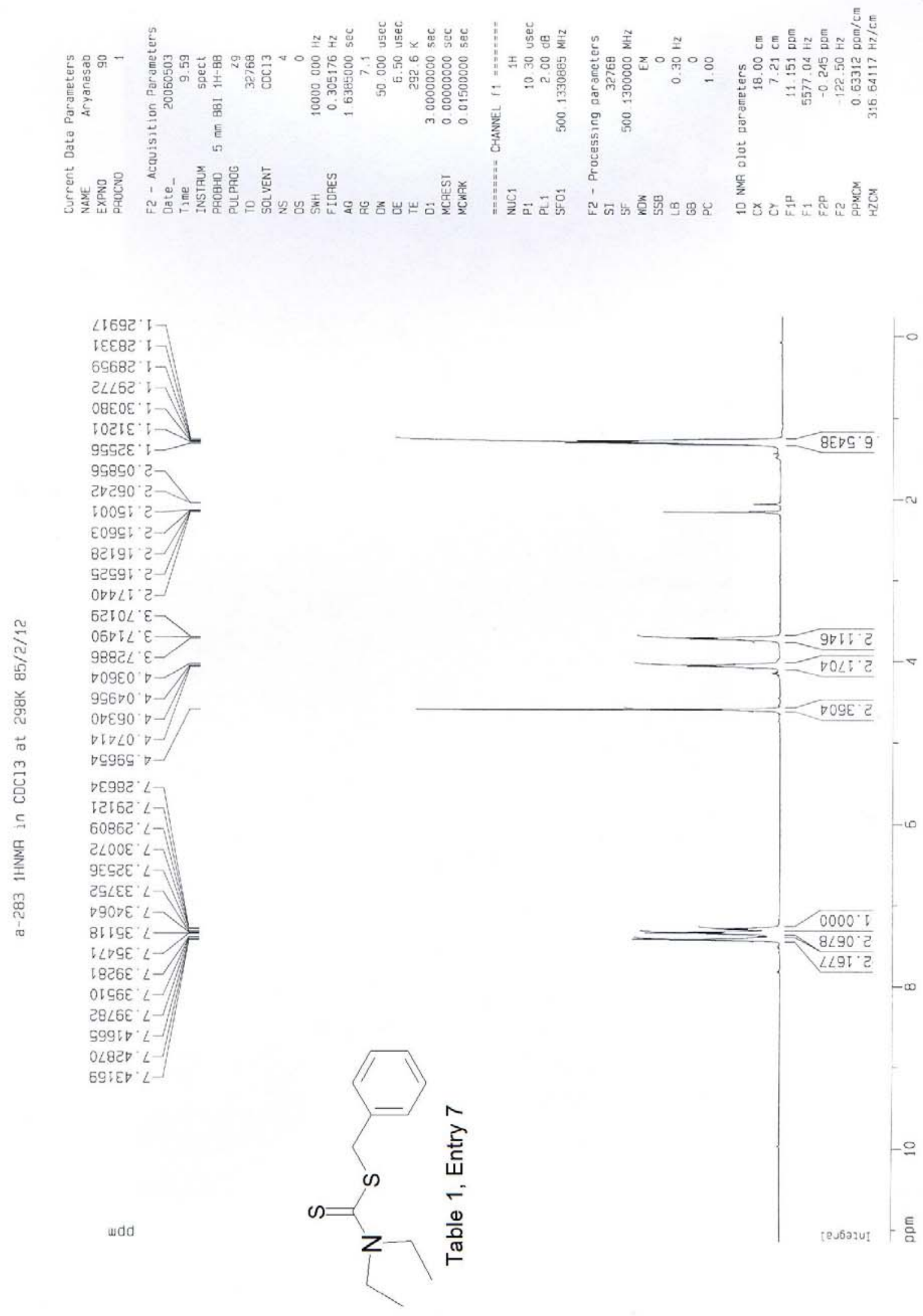


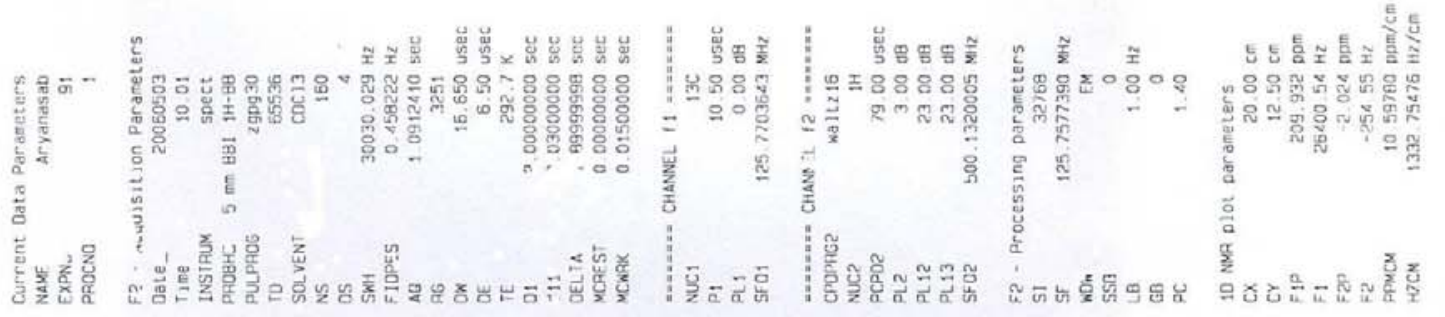

ટટઍ ટ

$\angle 20^{\circ} E$

EGE เE

989 27

$8 L^{\circ} 9$

$896^{\circ} 67$
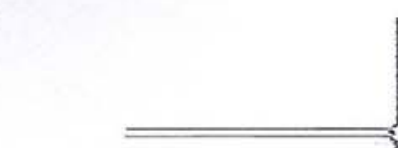

ते

SEL LL

$186^{\circ} \mathrm{LL} \longrightarrow$

gहट $8 L$

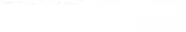

6I6 $\angle 21$ 698.821

$\nabla \angle 0^{\circ} 621$

$\nabla 6 \mathrm{I} 62 \mathrm{t}$

$\angle E B \cdot 62$ I

Oद9 $98 \mathrm{I}$

५५) ५6
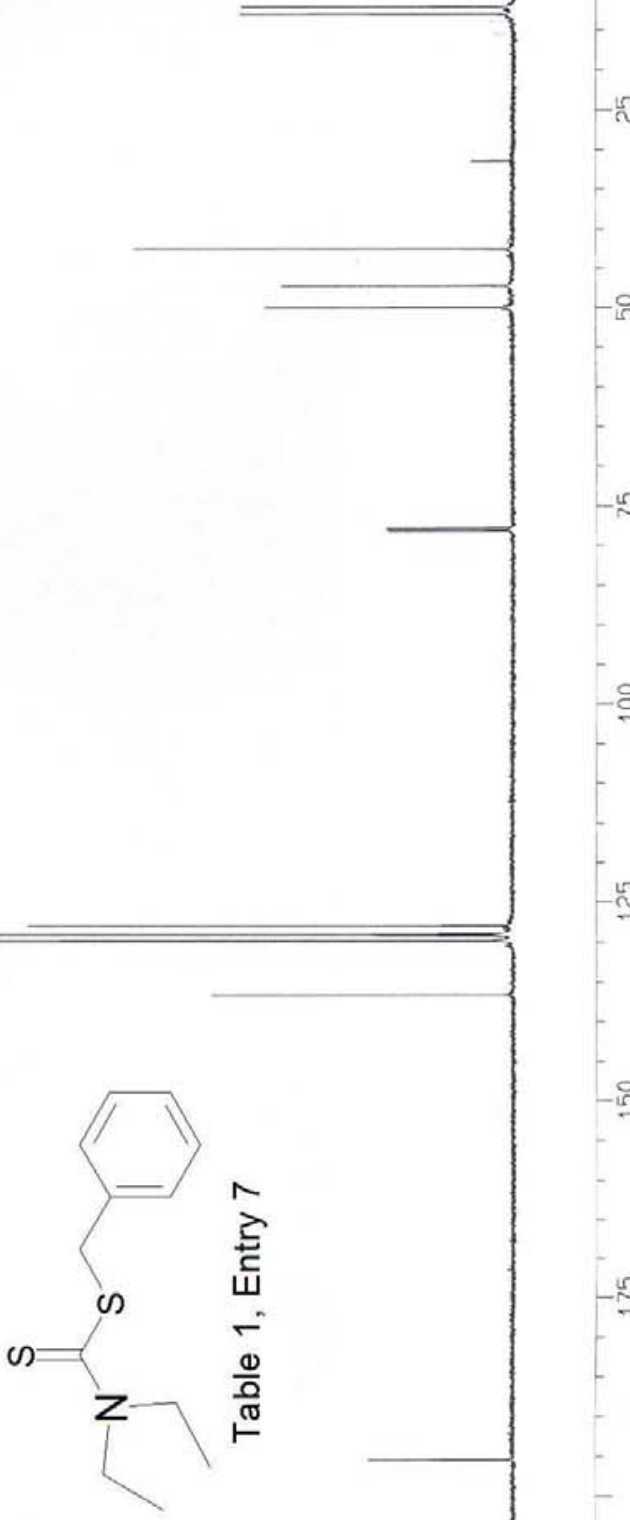

udd

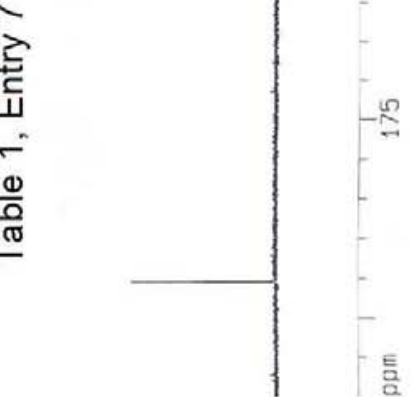




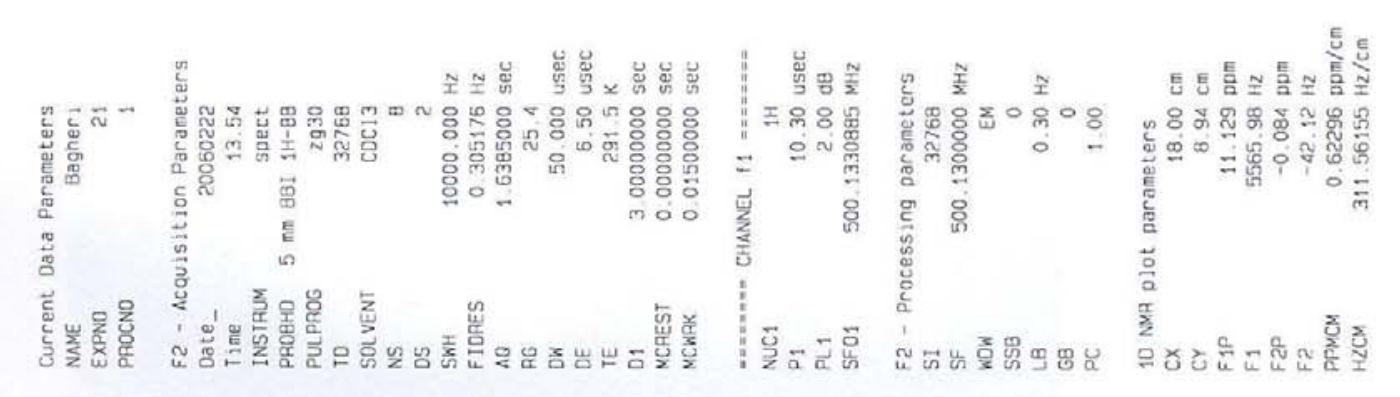

$80070^{\circ} 0$

2IG82

SCLOE

$\angle D$ IZE V

ह09єE :

LL29E' :-

$82980^{\circ} \mathrm{C}$

$909 \nabla \angle E$

$9265 \angle$ '

$\angle G E L L E-$

$8 \angle \angle 8 L L^{\circ}$

$85 \nabla 90^{\circ} \nabla$

$\angle E 890^{\circ} \circ$

$99280^{\circ} \mathrm{v}$

$99960^{\circ} \mathrm{b}$

ESOLG'

$\angle G 6 \angle G^{\circ} \nabla$

$66019^{\circ} \circ-$

92०92 $\angle 7$

9L992 $\angle 7$

$080<2^{\circ} \angle \neg$

$905 \angle C^{\circ} \angle$

เEเ $6 \mathrm{C}^{\circ} \mathrm{L}$

ट800E $\angle>$

टG90E ' $\angle$

9680E' $\angle$

ᄀ०टाह $\angle$

889tE' $L$

OEECE ' $L-$

$\checkmark I \angle Z E^{\prime} \angle-$

9EटEE ' $L$

$900 \vee \varepsilon^{\circ} \angle$

COBID $\angle$

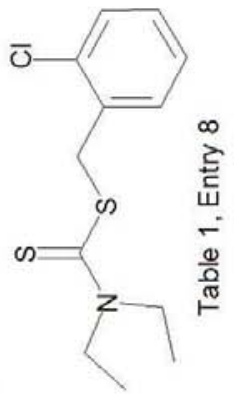

wdd

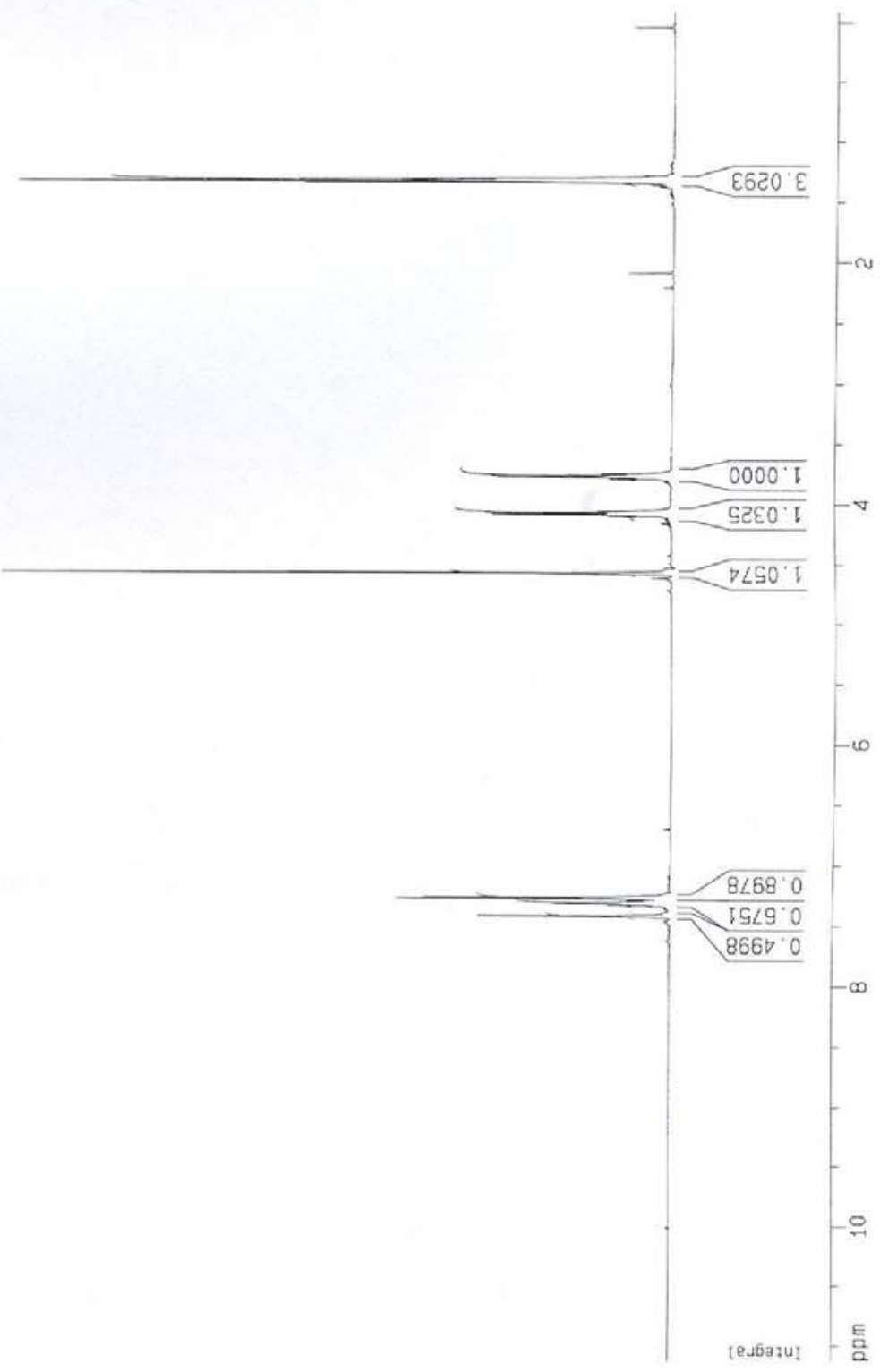




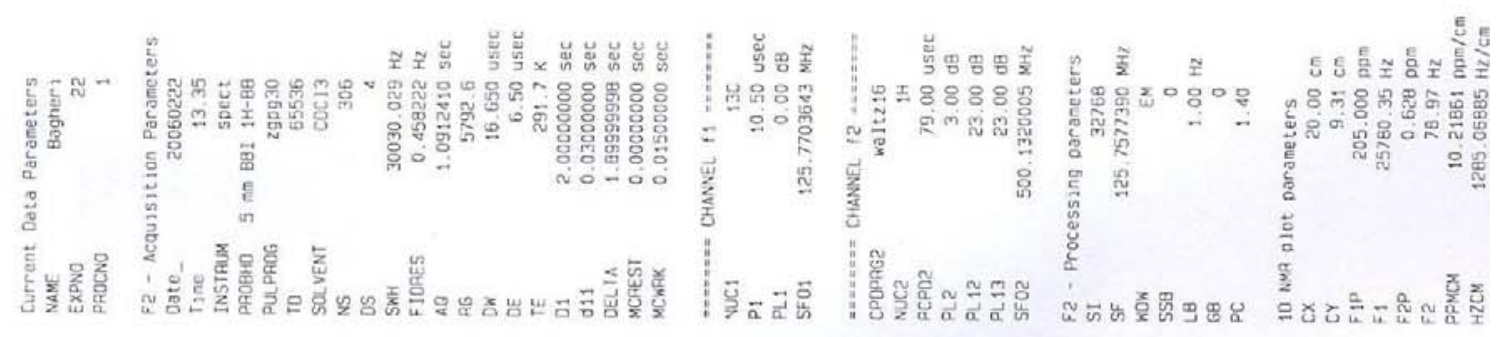

$620^{\circ}$ 己

086 ㄴ

IL it

$8 E 2 \angle V$

DEI OG

2OE' $\angle L$

$99 S^{\circ} L L$

1. $18^{\circ} \angle L$

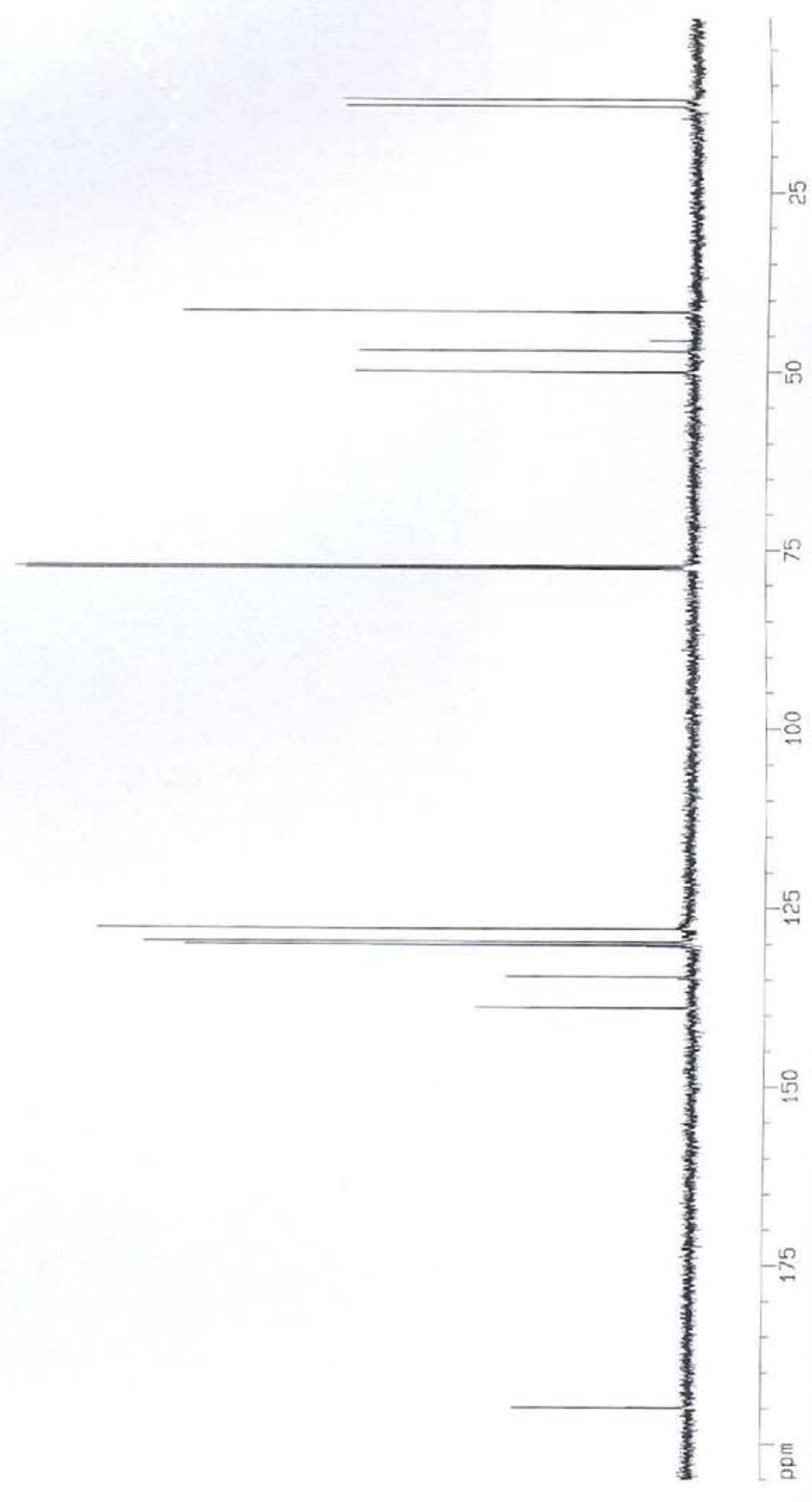

510.821

$\angle 66^{\circ} B \mathrm{~B} I$

16L Бटा

80ट OE

$\checkmark 99^{\circ} \nabla E L-$

टB6 $8 E$ I

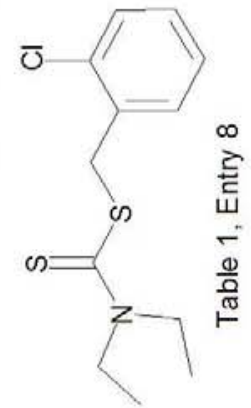

$066^{\circ} \nabla 67$

wdd 

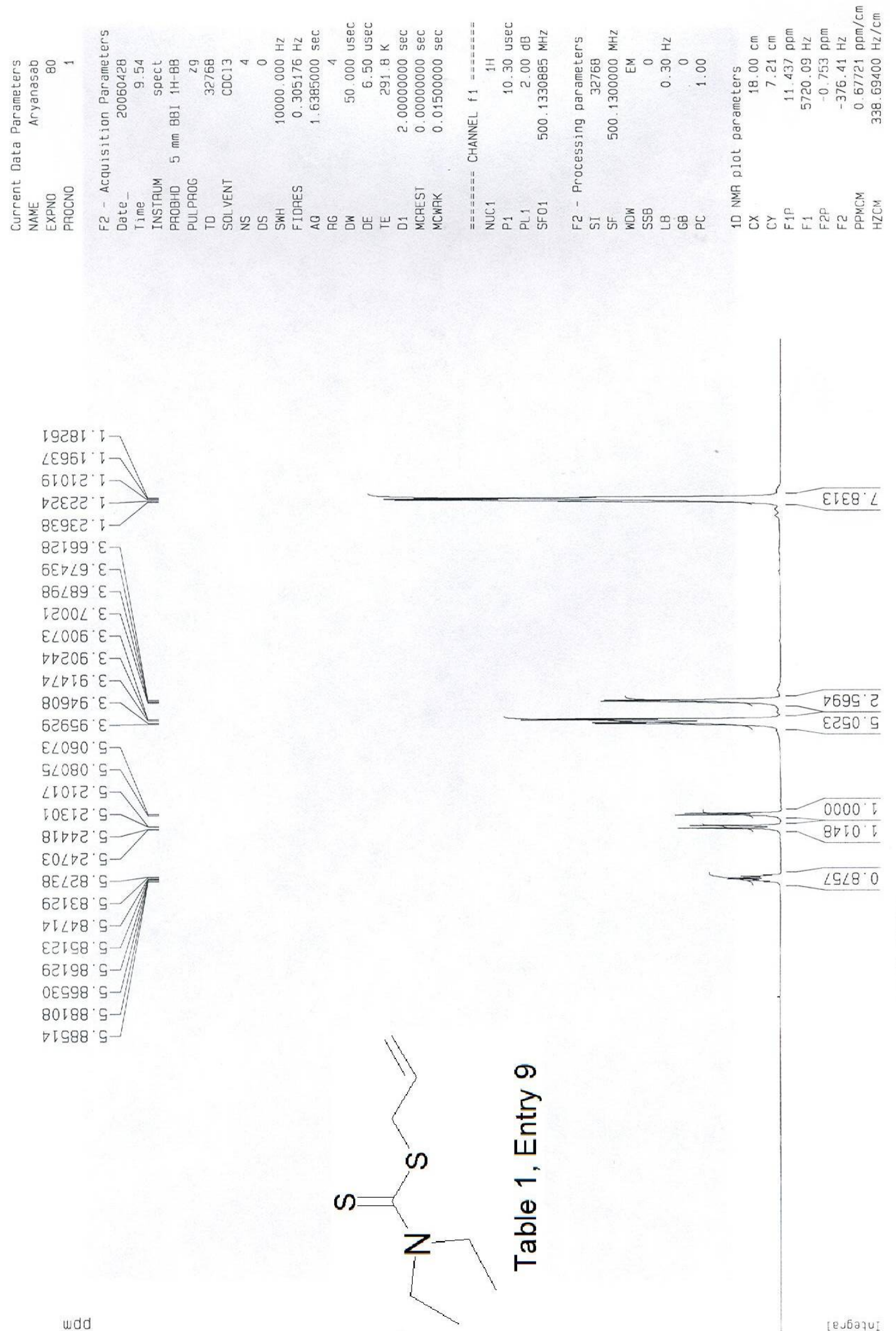


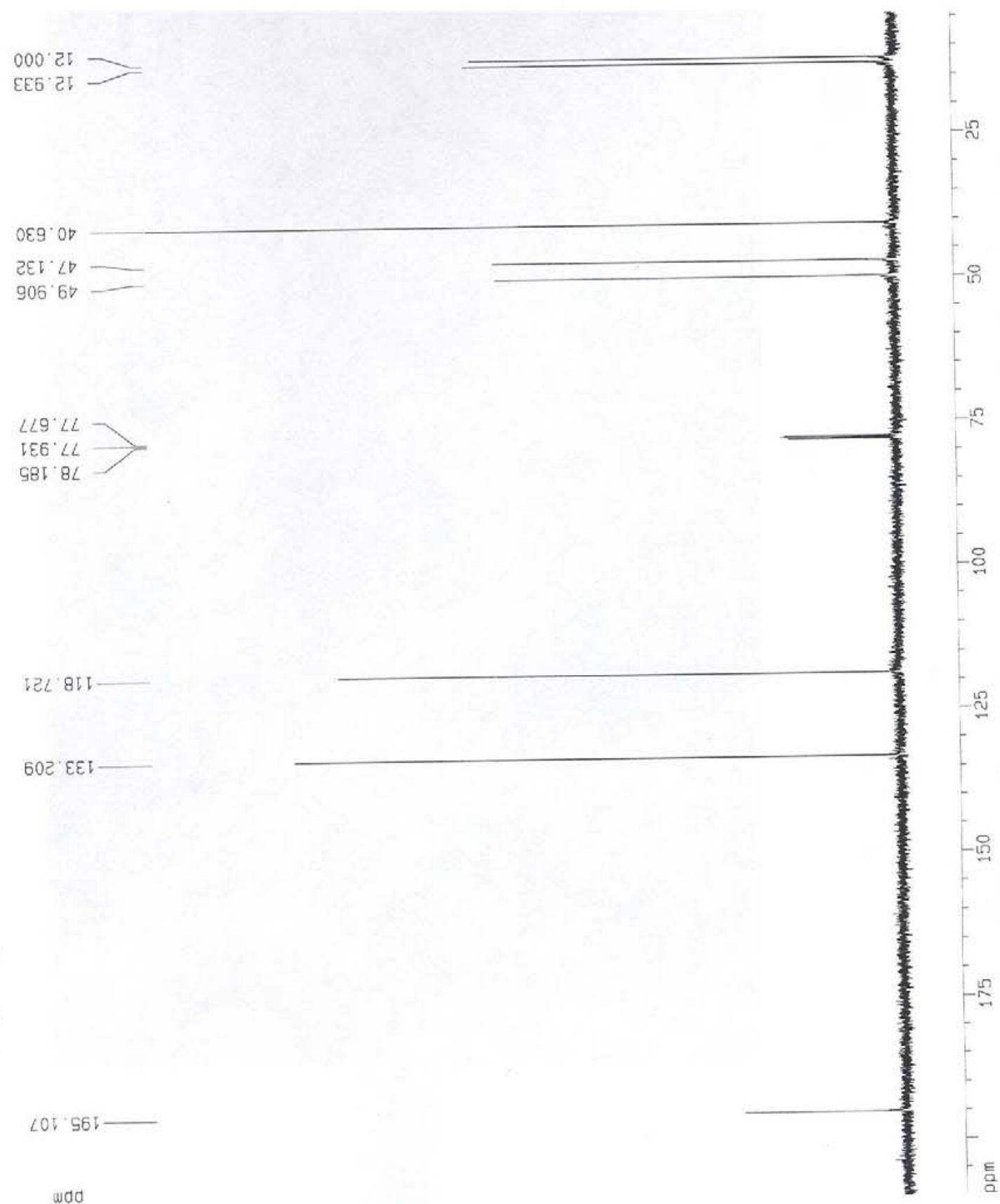



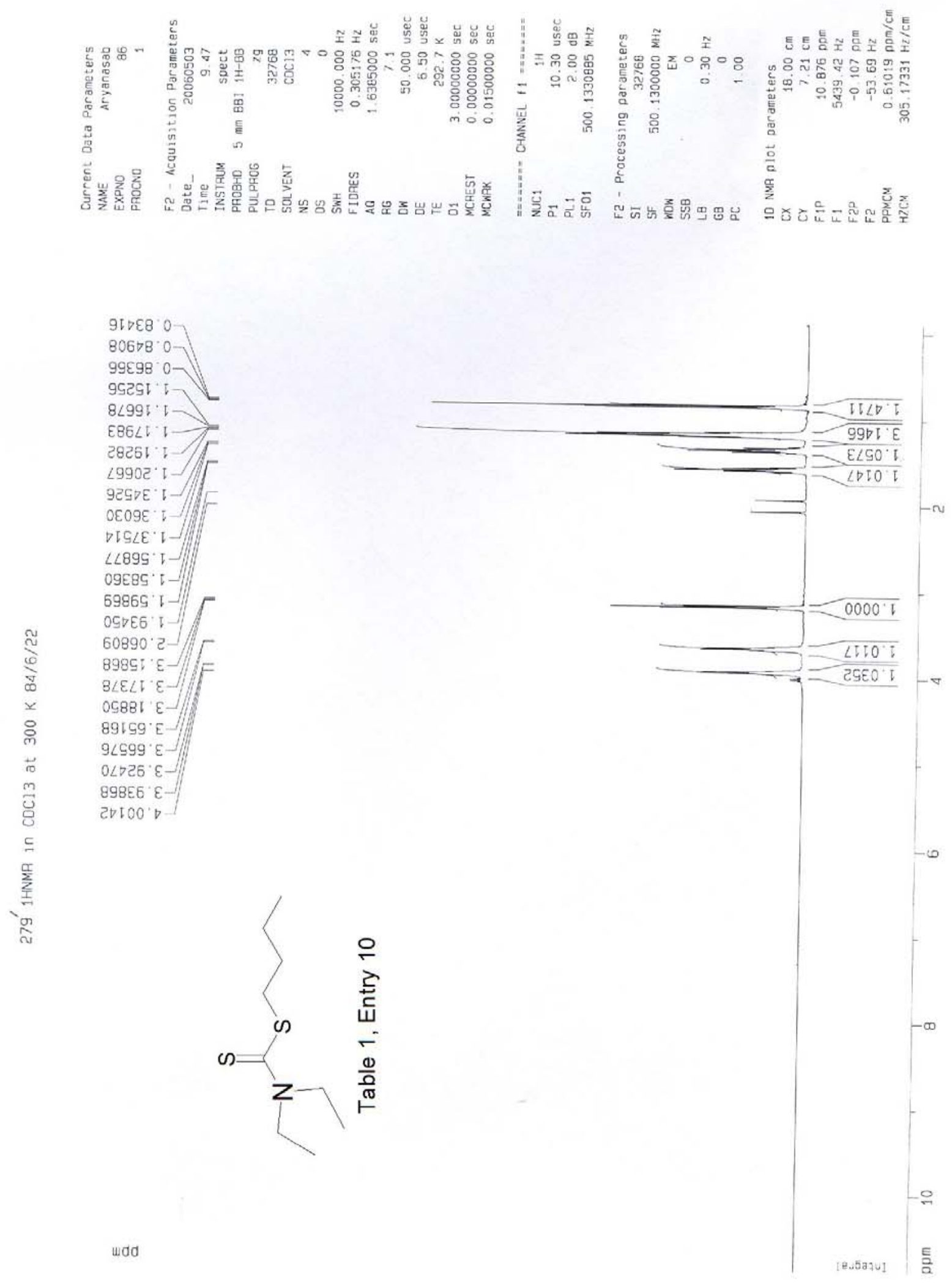


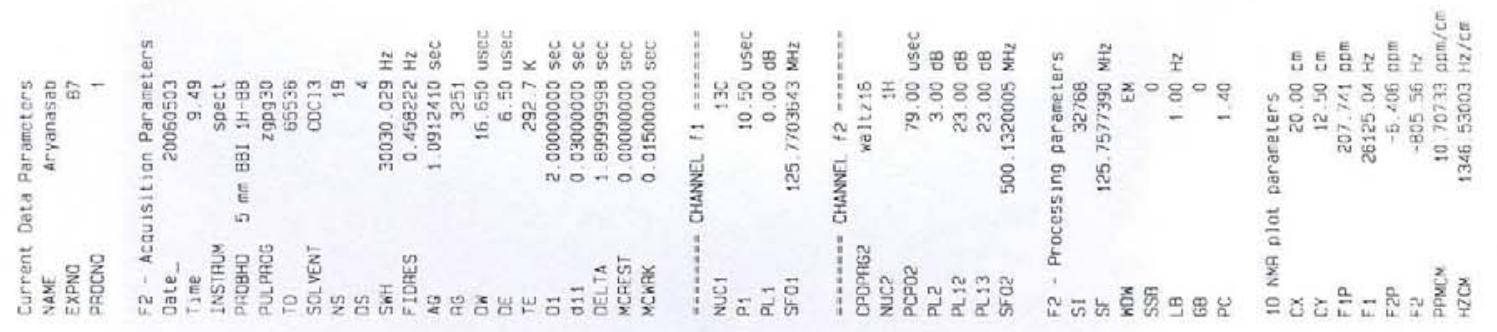

$\angle 96^{\circ}$ it

$98 \angle 21$

EOI $\nabla T-$

$\triangle E G^{\prime} 2 己$

DOT' IE

9 Iद IE $>-$

$\triangle O Z \angle E$

$\varepsilon \angle 6^{\circ} 97$

Sog $6 \mathrm{D}$

5.9.09
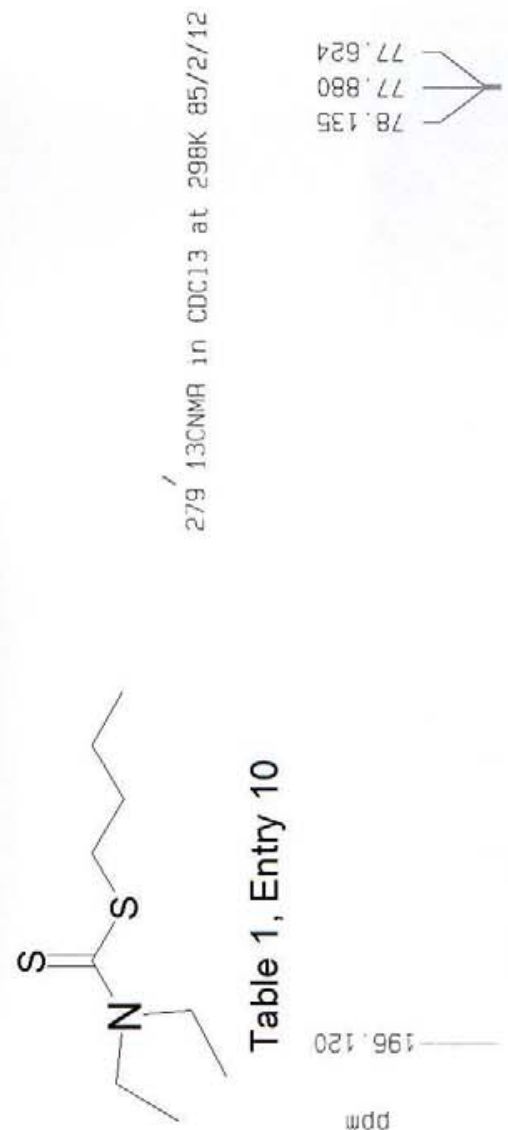

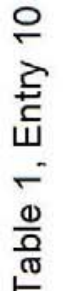

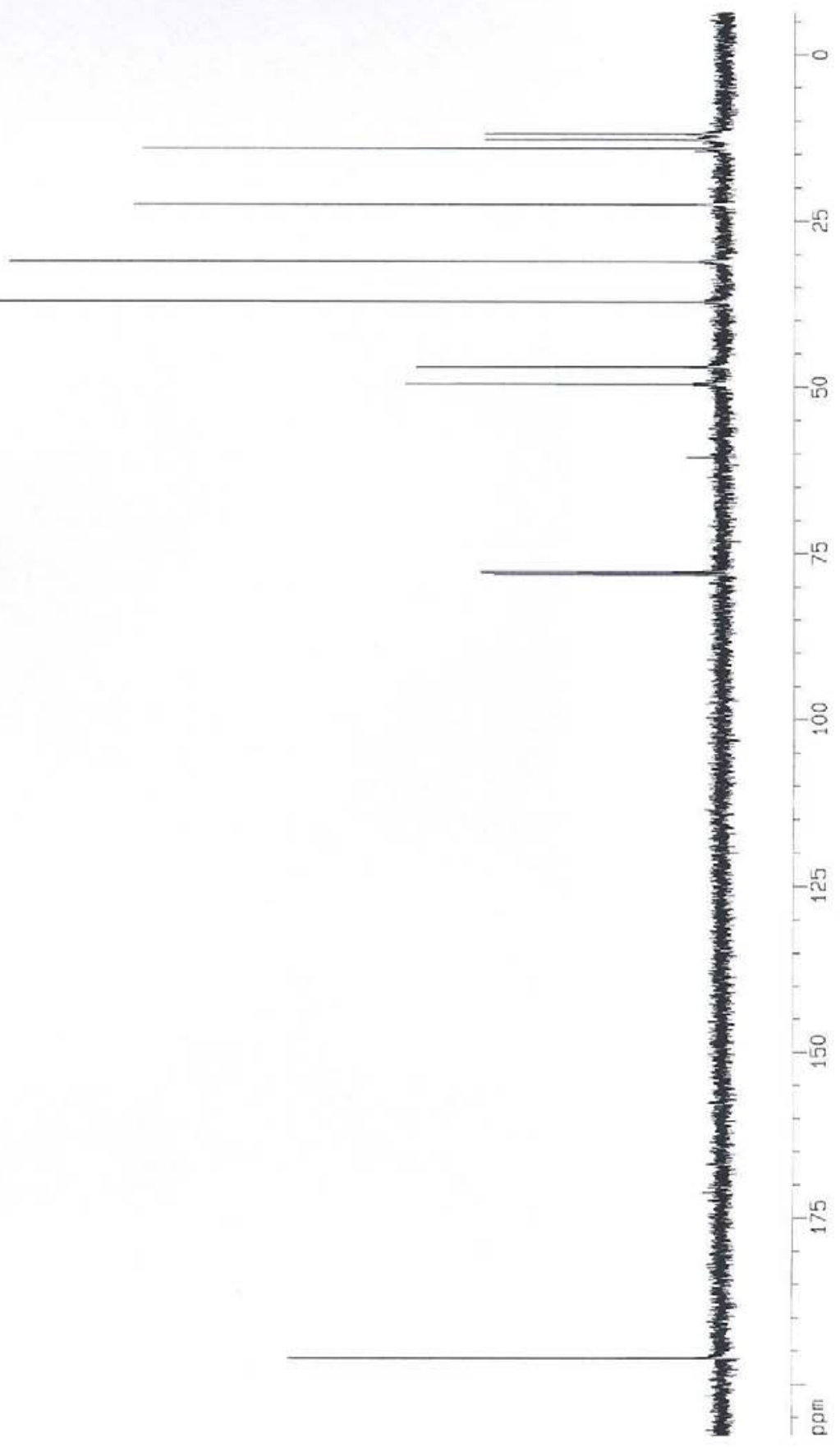



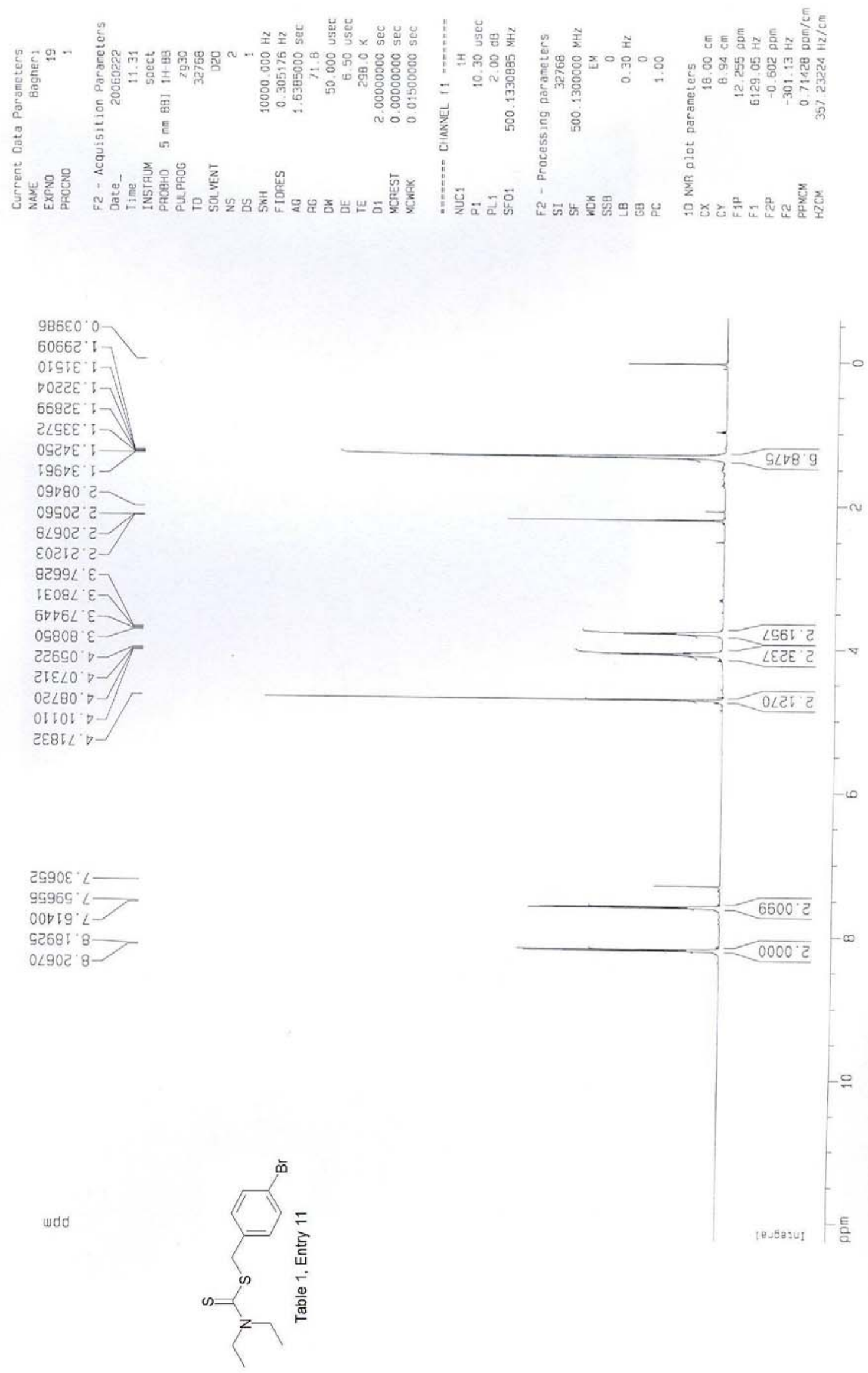


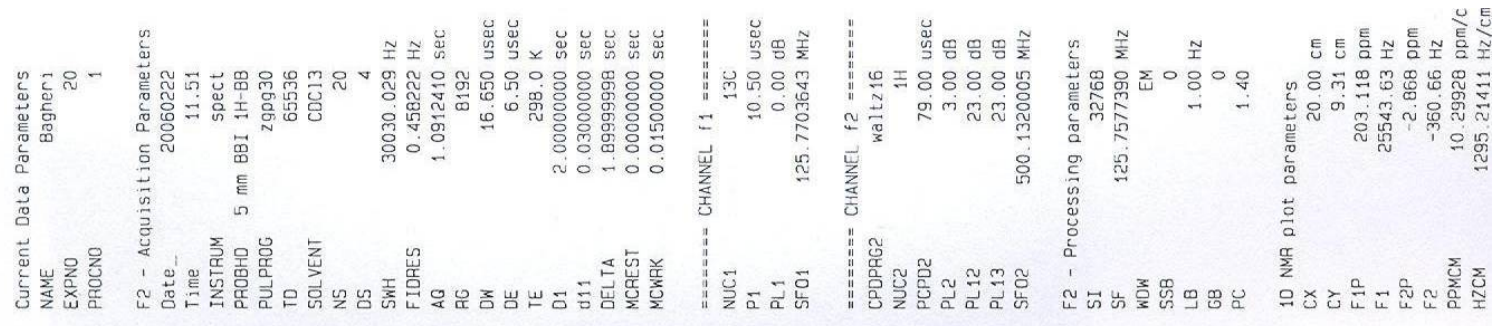

$\nabla 96^{\circ} \downarrow \tau \longrightarrow$
$\nabla 00^{\circ} \varepsilon \downarrow-$

296 0

$9 \nabla \varepsilon^{\circ} \angle$

¿9० OS

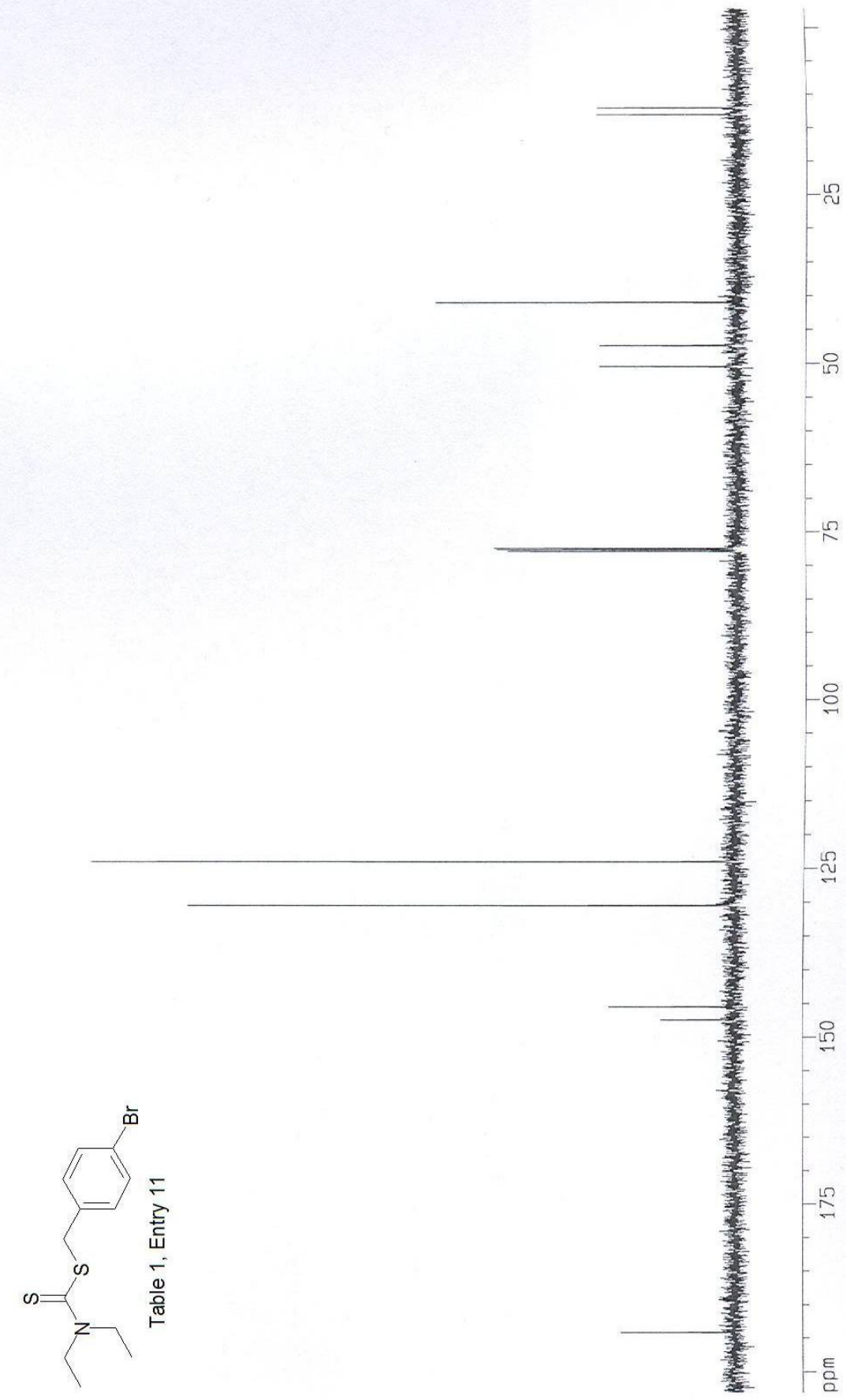

$\nabla \sigma \mathcal{E}^{\circ} \angle L$

$6 \nabla 9^{\circ} \angle L$

$206^{\circ} \angle L$

$020^{\circ} \nabla 2 T$

26॰ OE

$90 \mathcal{G}^{\circ}$ St

$6 S^{\circ} \angle D V$

ट๐レ จ6レ

udd 

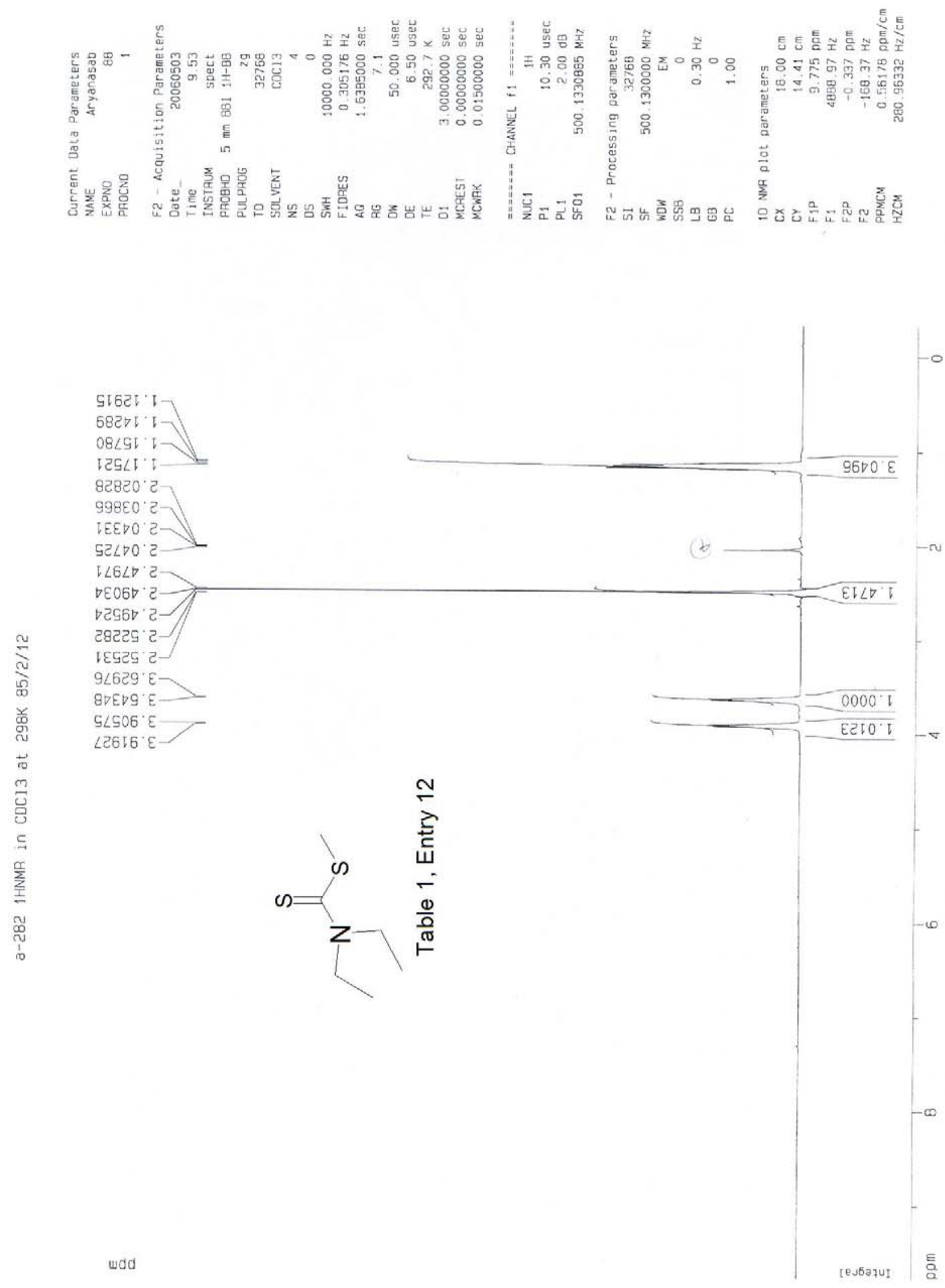

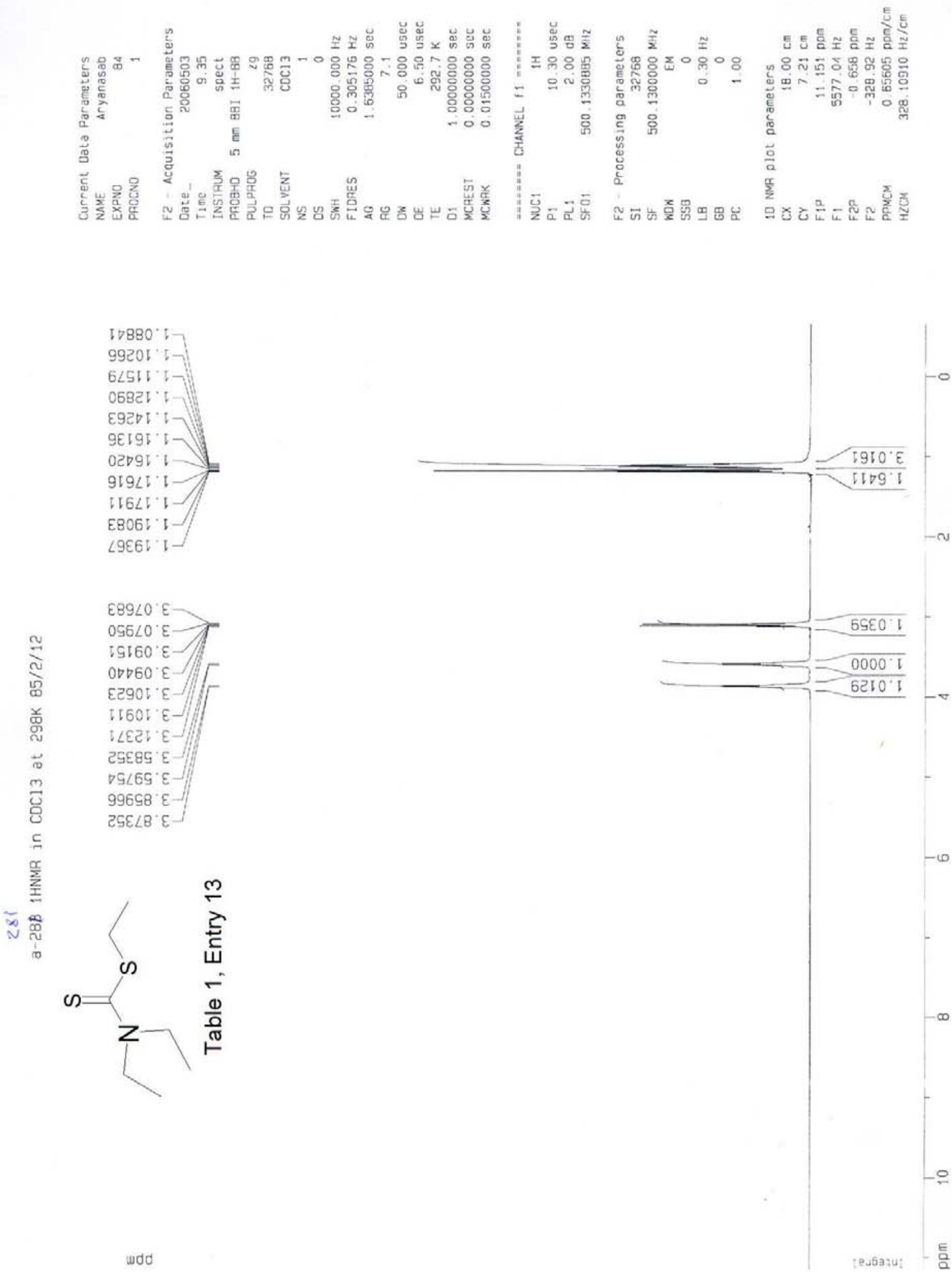

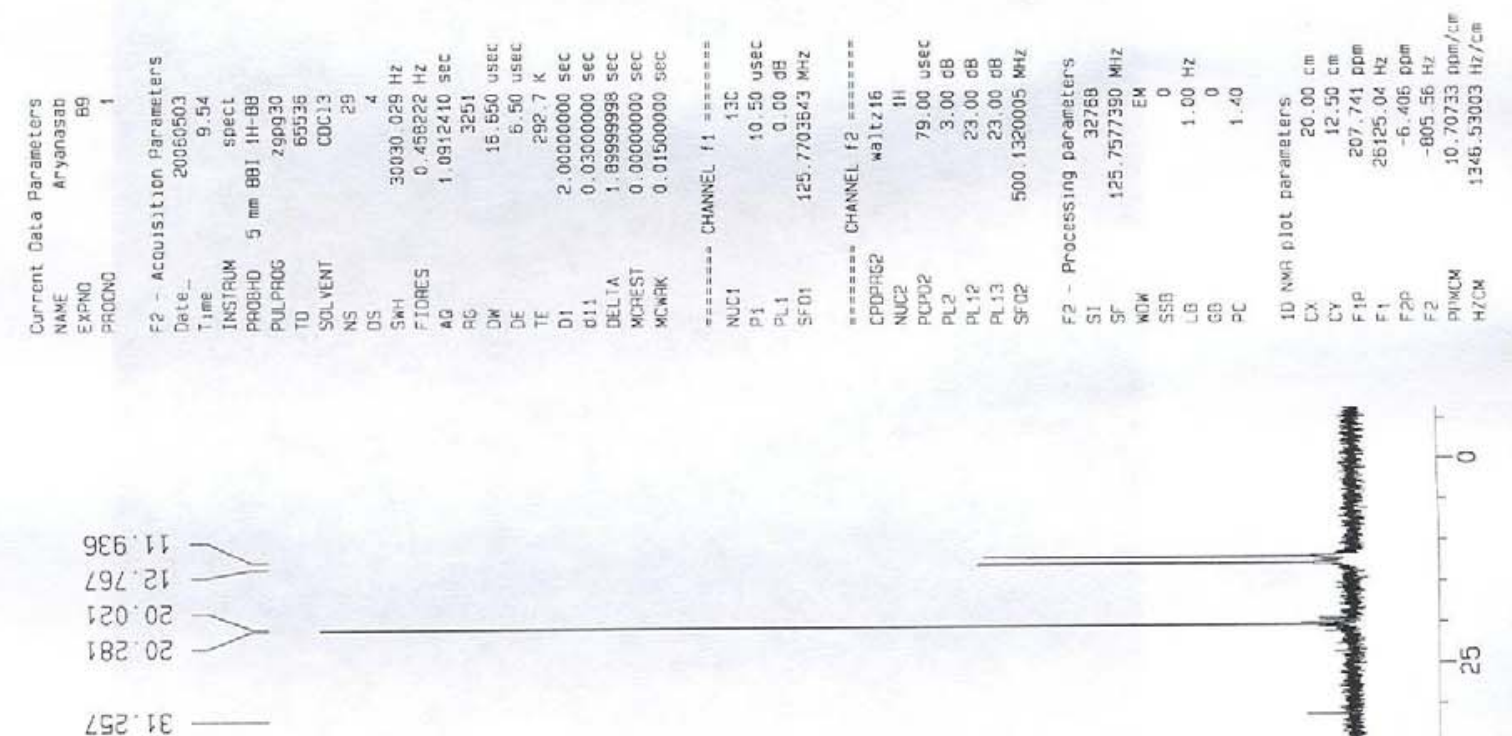

$500^{\circ} \angle D-$
$508^{\circ} 60$

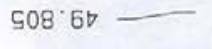

$\begin{aligned} & \angle V L \angle L \\ & 2008 L \\ & \angle 52 \cdot 8 L\end{aligned} \longrightarrow=$

लू

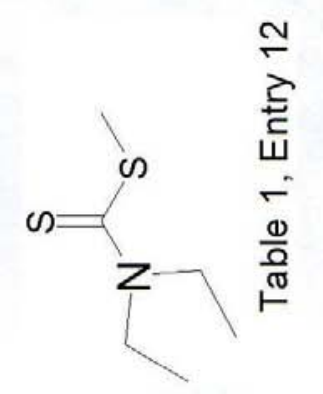

$\nabla 2 L 961$

udd

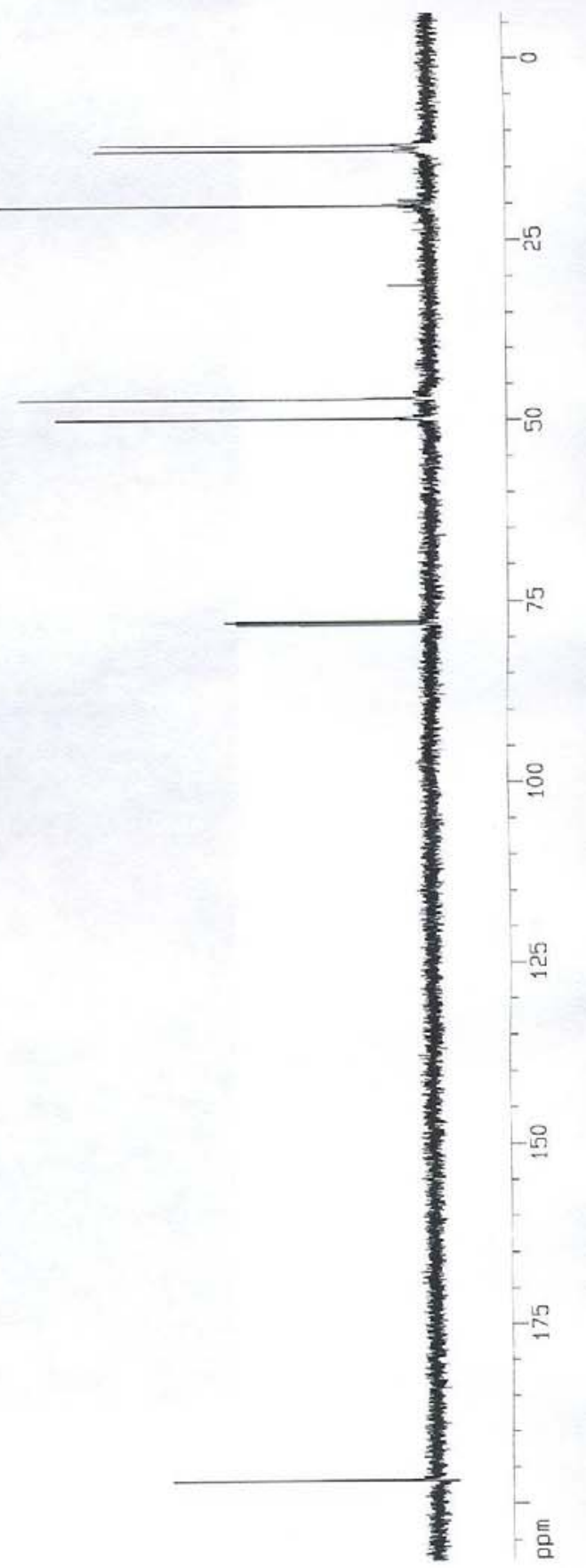




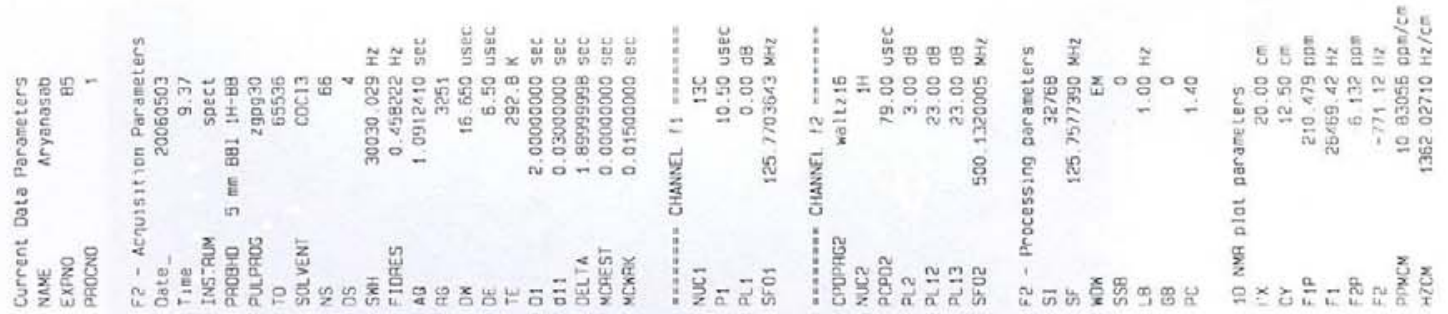

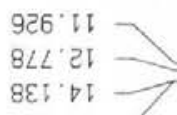

$\nabla G V^{\circ} \nabla t$

$\angle 99^{\circ}$ '

Eจ 97

$69 \nabla^{\circ} 6$

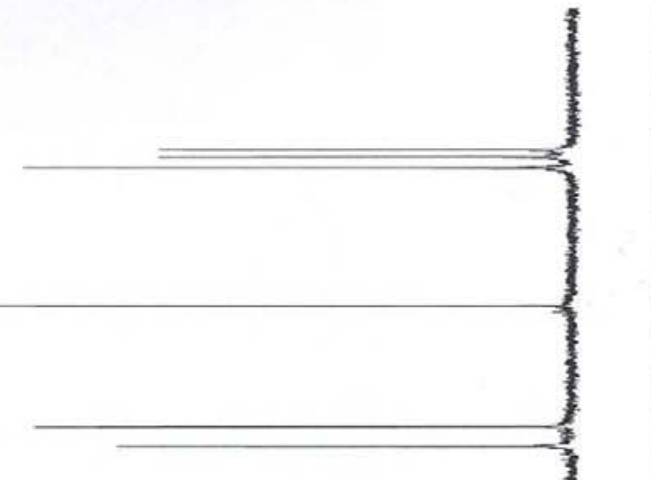

$96 L \angle L$

ISO $8 \angle$

GOE' $8 L$
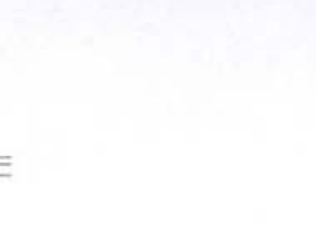

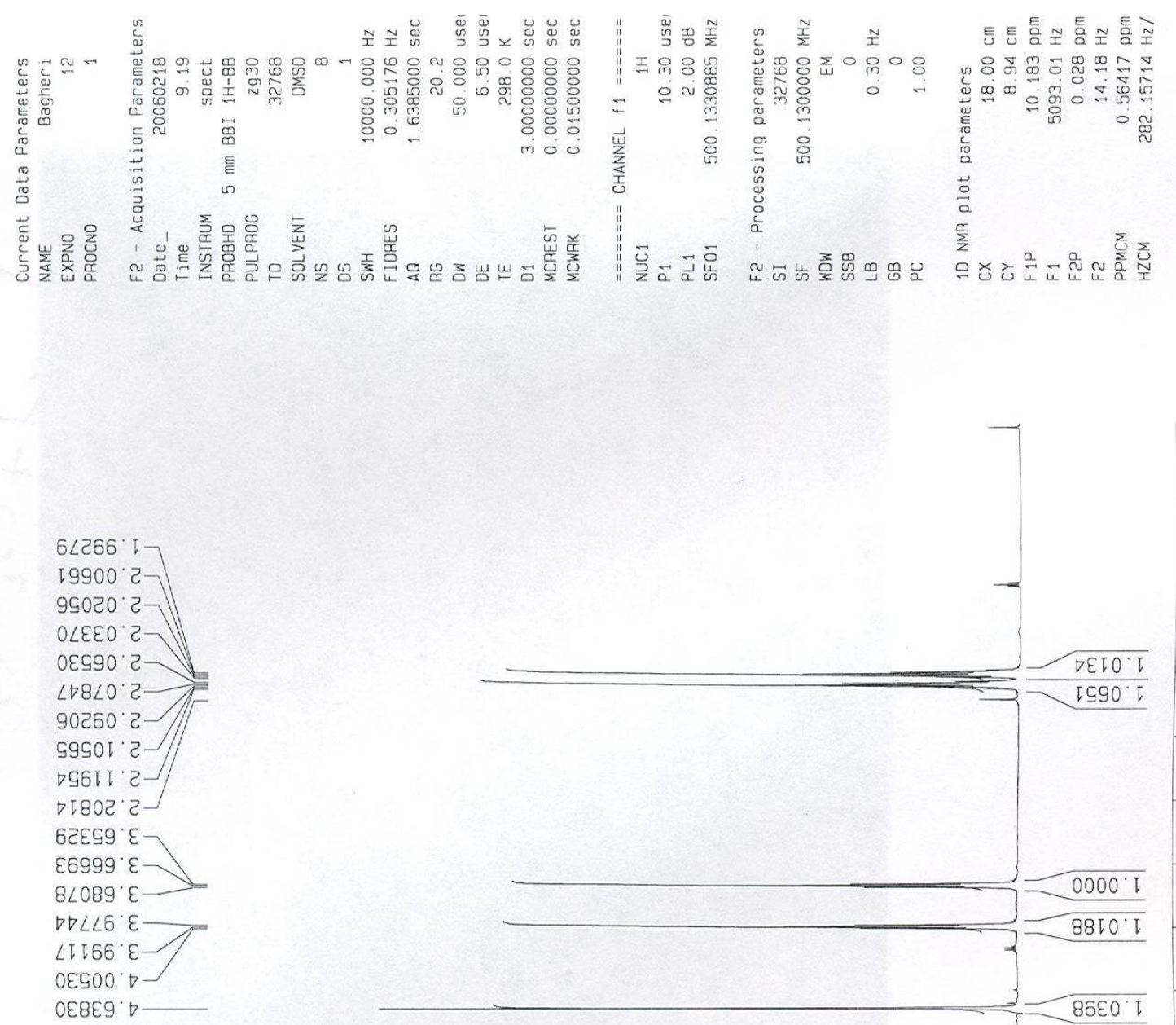

Бटट8ट $\angle$

98962 ' $L$

SटIOE $\angle \neg$

ट880E $\angle$

टDIV $L$

$\checkmark O \angle E E^{\circ} \angle$

896ฤE $\angle$

จEटGE $\angle$

GSE9E $\angle$

$\nabla 999 \varepsilon^{\circ} L$

$\varepsilon \angle \nabla E \nabla \nabla^{\circ} \angle$

996⿰习' $L$
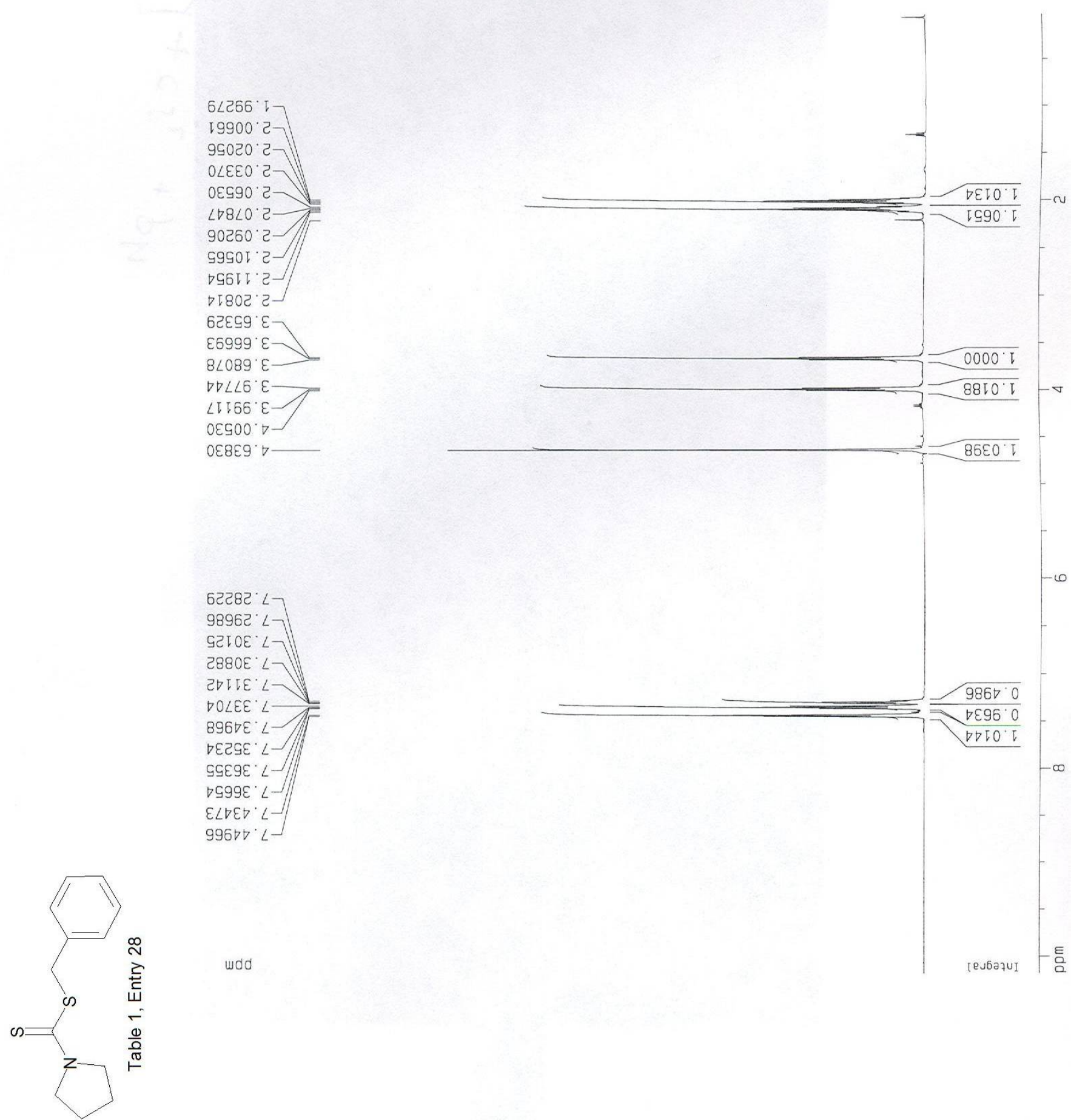

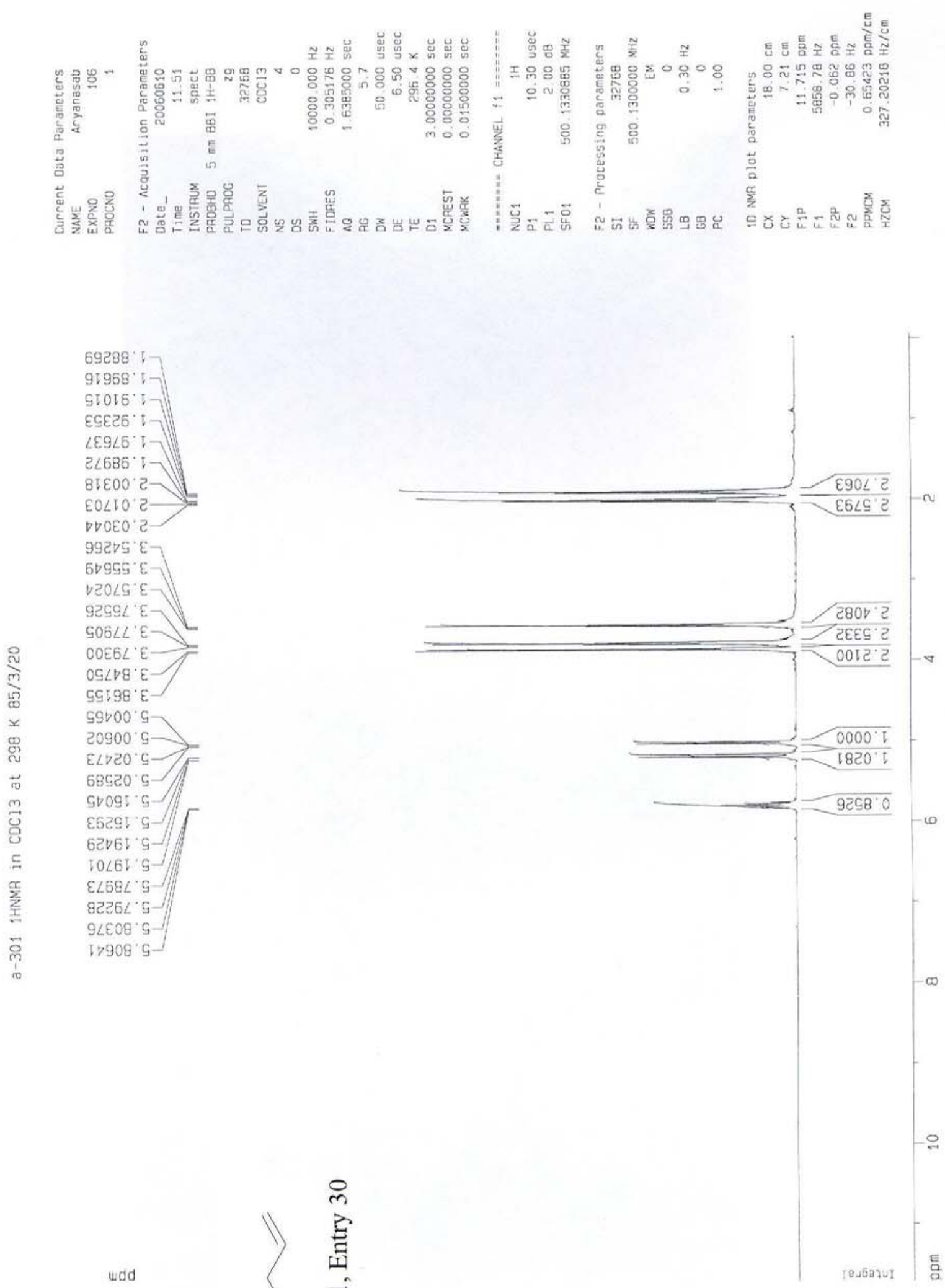

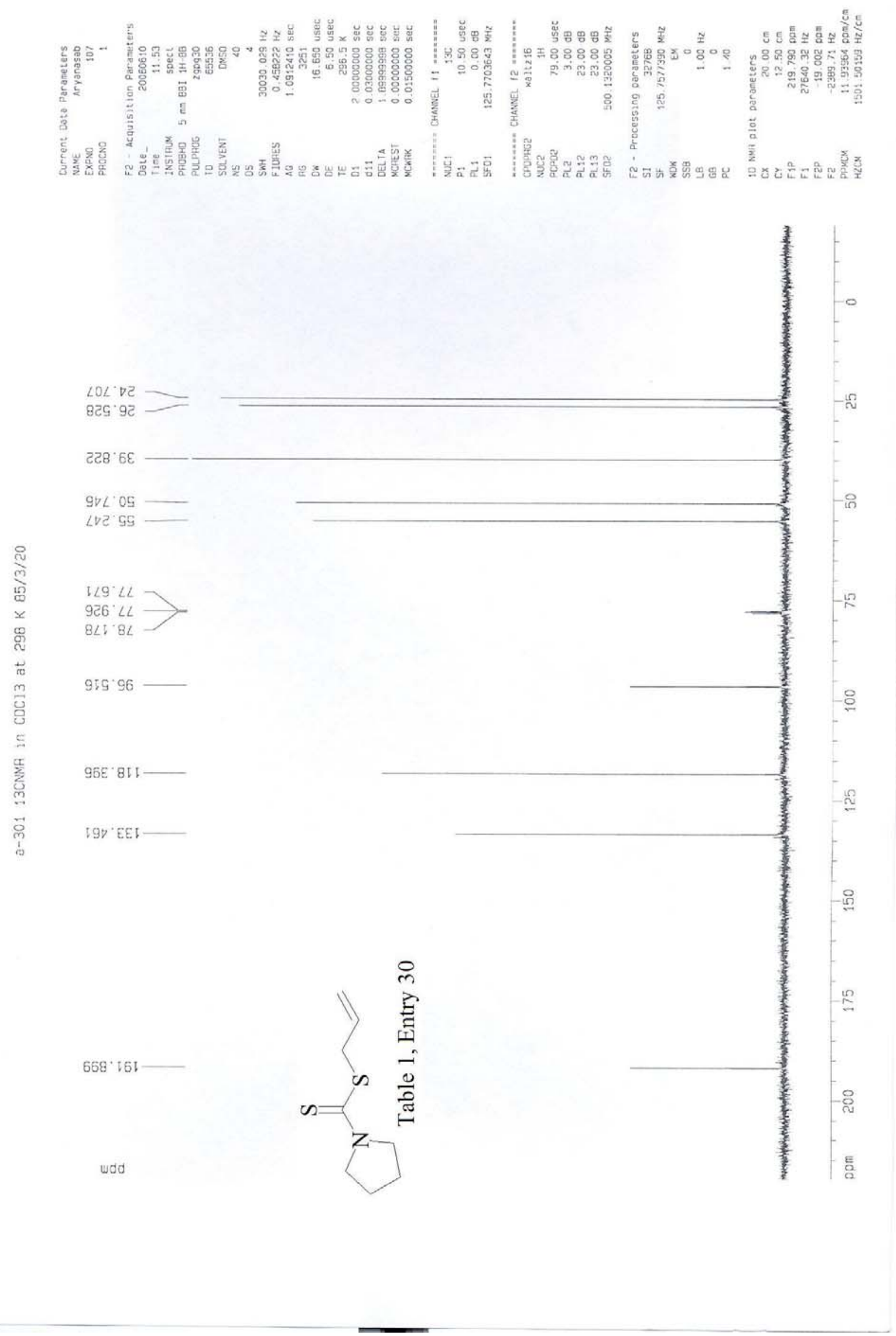

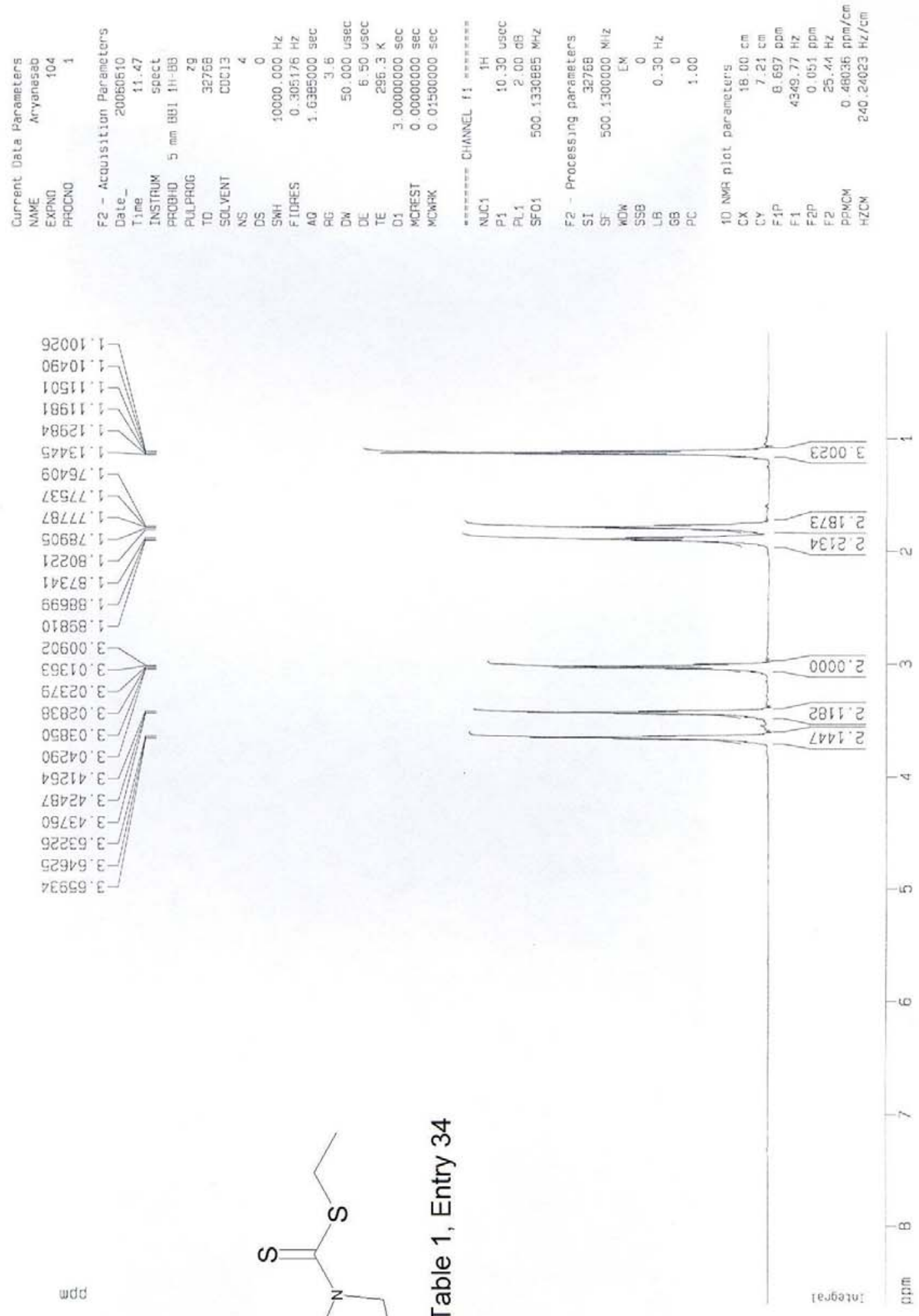


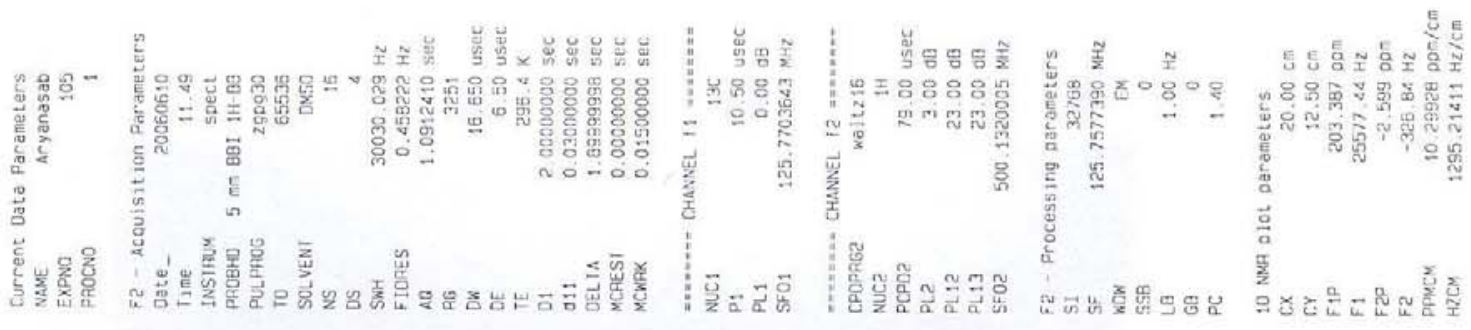

टटद $\forall 1$

$852^{\circ} \nabla \mathrm{c}$

SEb $\cdot 92$

TEL OE

$959^{\circ} 09^{\circ}$

$105^{\circ} \nabla 9$

$906^{\circ} \angle L$

ट9. 84

9.t. $8 \angle$

$\nabla 87 \cdot 96-$

뭉

돈

紊
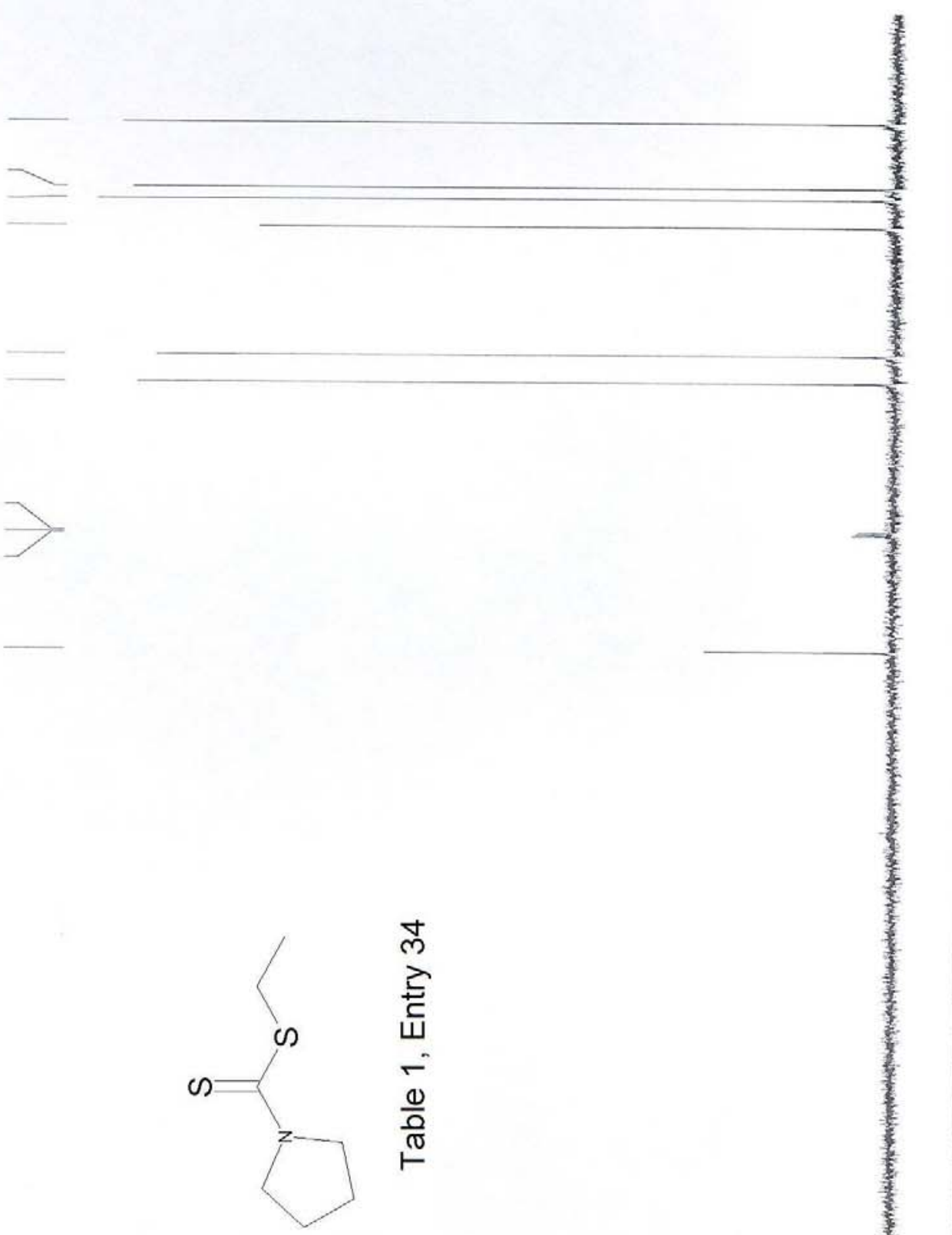

$58 E^{\circ} 261-$

wdd

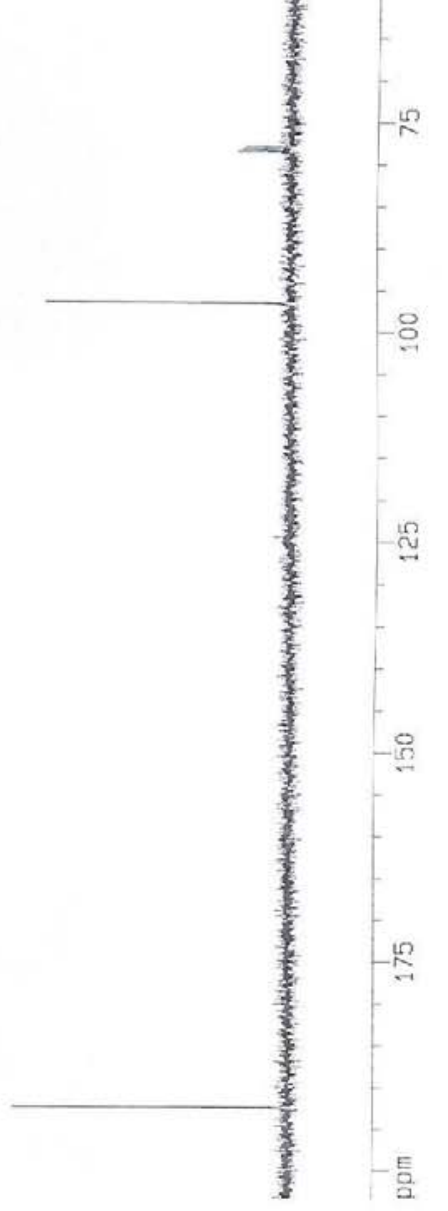



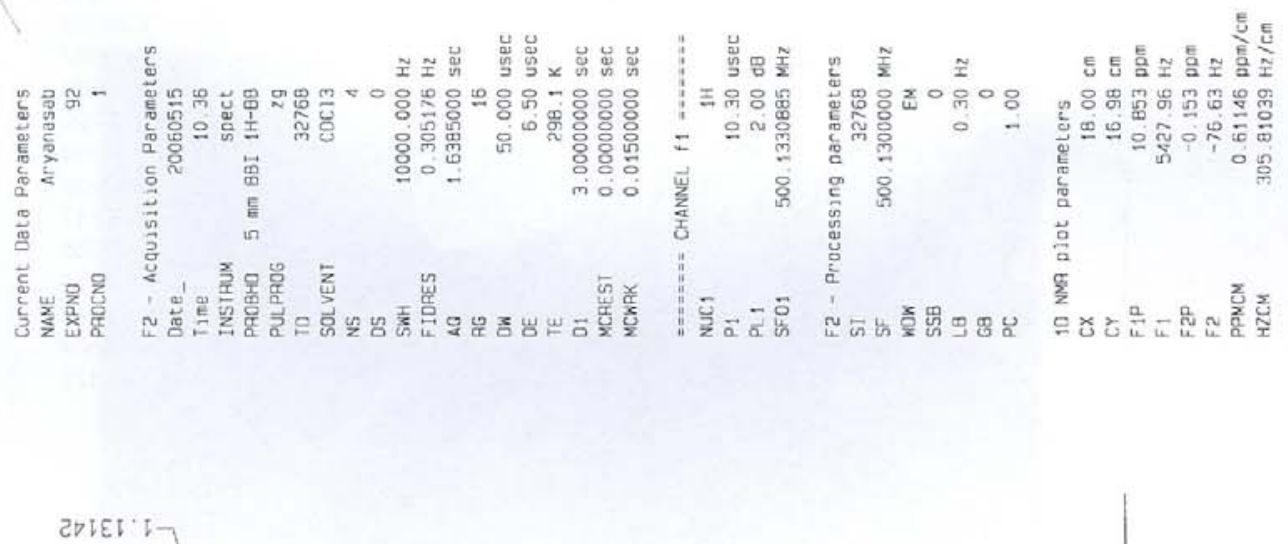

ELGDI '

6I69) ' 1 -

โटEOอ : -

$81 \angle t 21 \longrightarrow L$

IDIEC

DIELE :

GE9จ० ट

SEOSO द-

मटद्व $\varepsilon$

$67079^{\circ} \varepsilon$

$\nabla E I 9^{\circ} \varepsilon$

$88 \angle 99^{\prime} \varepsilon$

$\angle 1999^{\circ} E-$

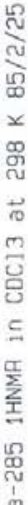

OL6 $\angle 9^{\prime} \mathrm{E}-$

$06269^{\circ} \mathrm{E}-\mathrm{C}=26 \mathrm{D} L^{\circ}$

$\angle 8 L L E-$

$\neg 9 \nabla G L E-A$

$622 \angle \theta^{\circ} E-$

E6588 ' $\varepsilon-$

$\angle B 668 \cdot \varepsilon-$

B9E เ6. $\varepsilon$

$980 \nabla 0 \circ-1$

BI $590^{\circ} \mathrm{D}$

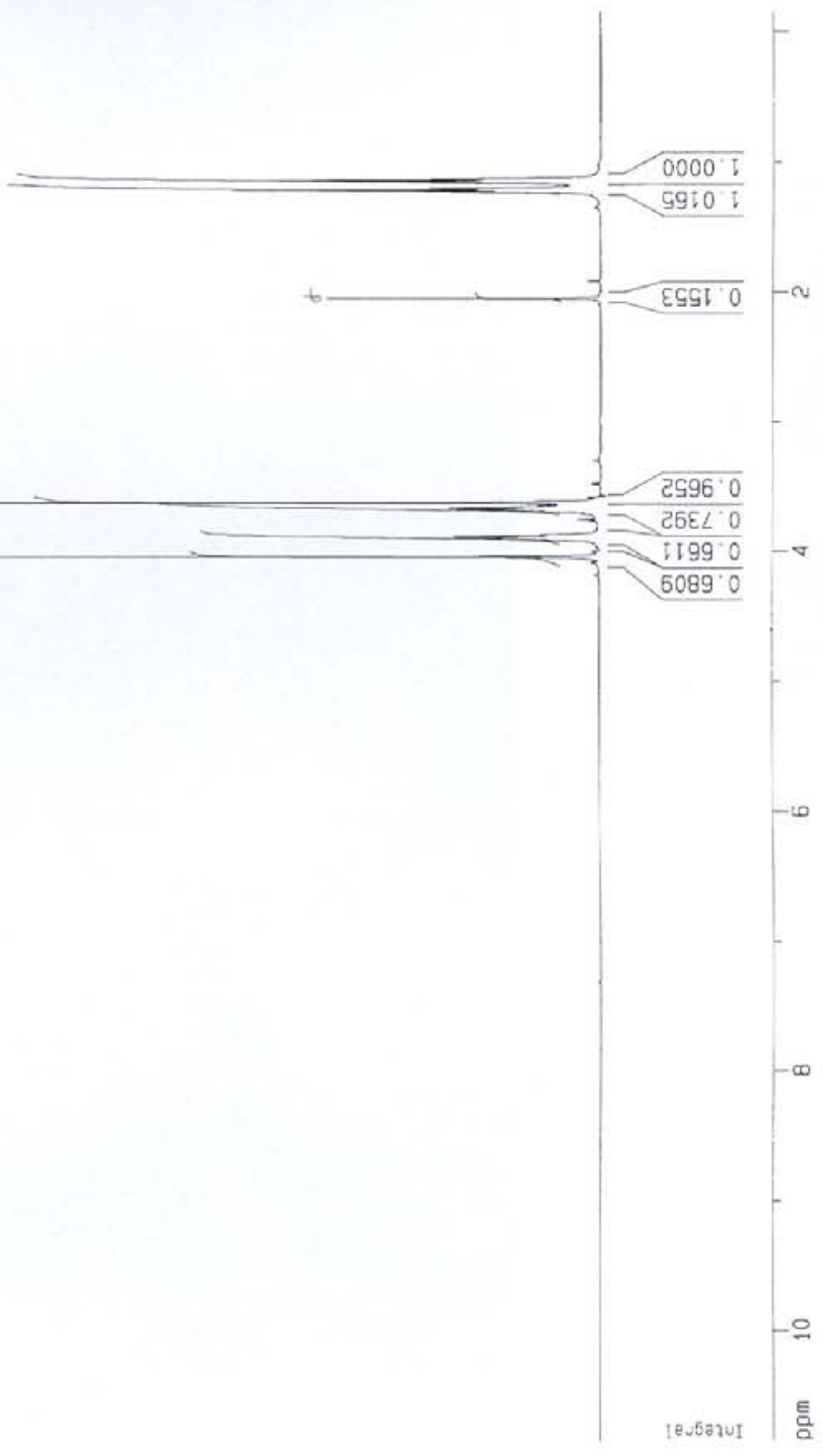



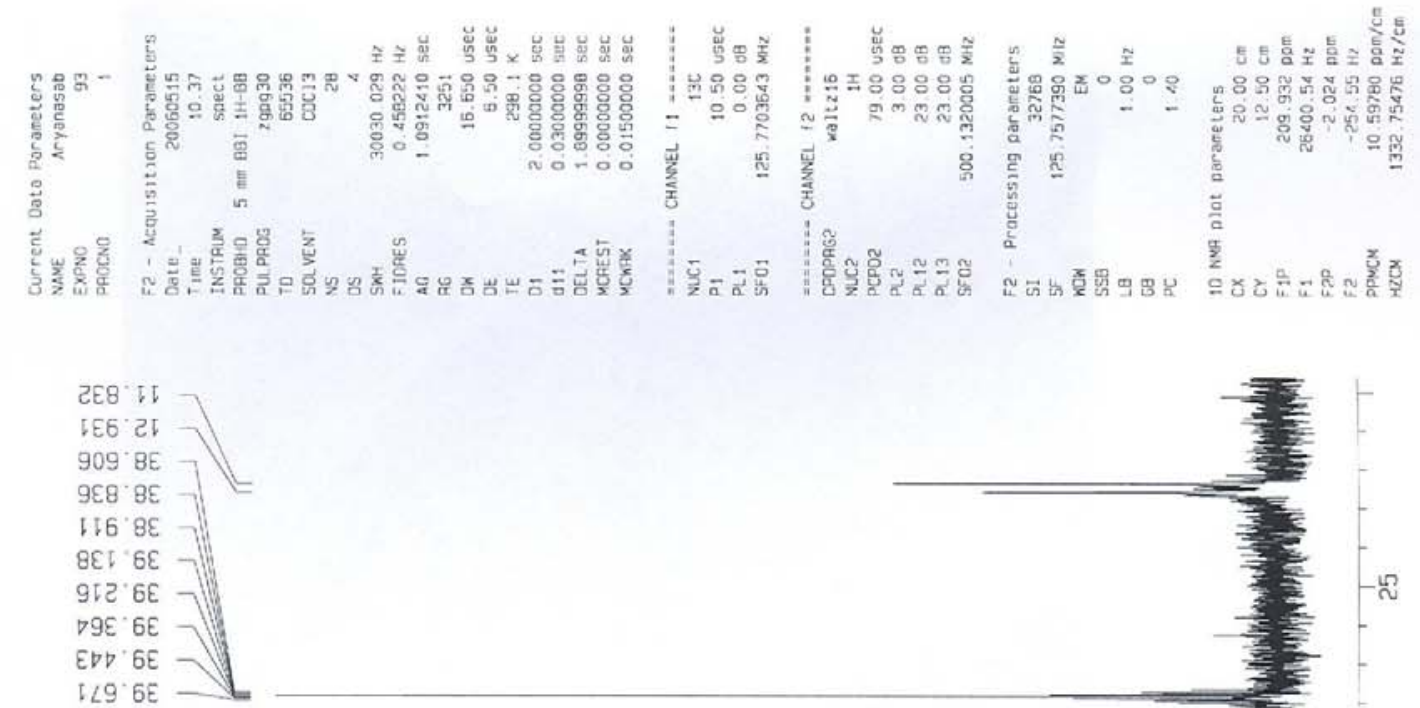

589.98

$59 \varepsilon^{\circ} \angle \mathrm{D}$

$898^{\circ} \angle D-/ F$
tटt $05-/ F$

562.09

I $90.09-$

$\triangle E D \cdot 25$

दटर टद

086.29 -

$0 \angle D^{\circ} \varepsilon$

$\left\lceil\angle 9^{\circ} \angle L\right.$

$926^{\circ} \angle L$

ह8I ' $8 L$
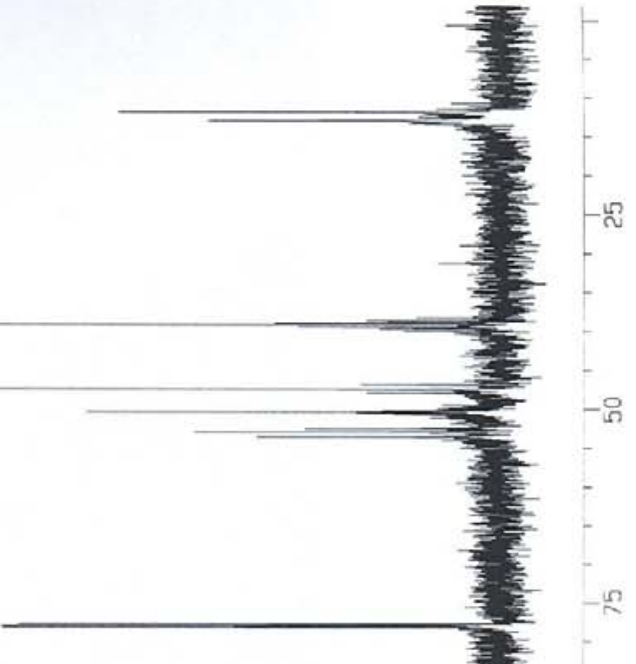